\title{
Three-dimensional flow of Newtonian and Boger fluids in square-square contractions
}

\author{
P.C. Sousa ${ }^{a}$, P.M. Coelho ${ }^{b}$, M.S.N. Oliveira ${ }^{a}$, M.A. Alves ${ }^{a, *}$ \\ a Departamento de Engenharia Química, CEFT, Faculdade de Engenharia da Universidade do Porto, Rua Dr. Roberto Frias, $4200-465$ Porto, Portugal \\ ${ }^{\mathrm{b}}$ Departamento de Engenharia Mecânica, CEFT, Faculdade de Engenharia da Universidade do Porto, Rua Dr. Roberto Frias, $4200-465$ Porto, Portugal
}

\section{A R T I C L E I N F O}

\section{Article history:}

Received 4 October 2008

Received in revised form 22 March 2009

Accepted 23 March 2009

\section{Keywords:}

Viscoelastic fluid

PIV

Visualization

3D contraction flow

Boger fluid

\begin{abstract}
A B S T R A C T
The flow of a Newtonian fluid and a Boger fluid through sudden square-square contractions was investigated experimentally aiming to characterize the flow and provide quantitative data for benchmarking in a complex three-dimensional flow. Visualizations of the flow patterns were undertaken using streakline photography, detailed velocity field measurements were conducted using particle image velocimetry (PIV) and pressure drop measurements were performed in various geometries with different contraction ratios. For the Newtonian fluid, the experimental results are compared with numerical simulations performed using a finite volume method, and excellent agreement is found for the range of Reynolds number tested $\left(R e_{2} \leq 23\right)$. For the viscoelastic case, recirculations are still present upstream of the contraction but we also observe other complex flow patterns that are dependent on contraction ratio (CR) and Deborah number $\left(D e_{2}\right)$ for the range of conditions studied: $C R=2.4,4,8,12$ and $D e_{2} \leq 150$. For low contraction ratios strong divergent flow is observed upstream of the contraction, whereas for high contraction ratios there is no upstream divergent flow, except in the vicinity of the re-entrant corner where a localized atypical divergent flow is observed. For all contraction ratios studied, at sufficiently high Deborah numbers, strong elastic vortex enhancement upstream of the contraction is observed, which leads to the onset of a periodic complex flow at higher flow rates. The vortices observed under steady flow are not closed, and fluid elasticity was found to modify the flow direction within the recirculations as compared to that found for Newtonian fluids. The entry pressure drop, quantified using a Couette correction, was found to increase with the Deborah number for the higher contraction ratios.
\end{abstract}

(c) 2009 Elsevier B.V. All rights reserved.

\section{Introduction}

The study of entry-flow problems, in which a fluid flowing through a duct of large cross-section progresses through a contraction into a smaller one, has been the subject of numerous studies with Newtonian and viscoelastic fluids [1-6]. Besides its great usefulness for understanding a variety of real-flow phenomena that exists in many engineering applications, such as extrusion and injection molding processes, it is also a classic benchmark flow problem used in computational rheology [7,8]. The majority of experimental and numerical works found in the literature on the subject of contraction flows focuses on axisymmetric and/or planar (or quasi-planar) arrangements. Planar flow configurations are particularly amenable for comparison with numerical predictions, since usually three-dimensional (3D) effects are negligible and experimental results can be adequately described using com-

\footnotetext{
* Corresponding author. Fax: +351 225081449.

E-mail addresses: psousa@fe.up.pt (P.C. Sousa), pmc@fe.up.pt (P.M. Coelho), monica.oliveira@fe.up.pt (M.S.N. Oliveira), mmalves@fe.up.pt (M.A. Alves).
}

putationally less demanding two-dimensional (2D) simulations. Axisymmetric contractions are ubiquitous in real-flow problems, and under steady flow conditions are also less demanding than full 3D simulations. Numerical research on 3D sudden contractions is less frequent since these require much more powerful computational resources. However, in some cases of interest, e.g. in microfluidic contraction geometries, the flow is markedly threedimensional due to reduced characteristic aspect ratios [9-11]. In such cases, a simple 2D approach is often qualitatively inadequate to describe the flow [12]. Contractions with significant 3D effects are not only interesting from a fundamental point of view, but are also useful for validating 3D numerical codes. As such, the square-square geometry, in which the contraction occurs in two perpendicular directions, is a candidate for a reference 3D test-case, since it offers a good compromise between geometric simplicity and complex 3D flow structure.

Entry flows through square-square contractions (or analogous configurations) have been documented only in a handful of papers, which have focused mostly on the flow patterns [13-20]. Some similarities between the flow through square-square and circular contractions were reported both in terms of the actual flow and 
in terms of the variation of the strain rates [19-21]. Most experimental and numerical studies typically report results concerning a $4: 1$ contraction ratio $(C R)$ in both directions (although $C R=13.3$ has also been considered in Ref. [21]). Indeed, this is motivated by the fact that the $4: 1$ contraction ratio was chosen as a benchmark testcase during the 5th International Workshop on Numerical Methods in non-Newtonian flows [7]. As a result, the flow patterns in this particular configuration are well documented for Newtonian and Boger fluids $[14,15,19]$. The flow of viscoelastic fluids through this type of contraction presents a rich variety of flow structures spanning a number of regimes, such as lip vortex activity, vortex growth, diverging flow and unstable flow. Despite these advances, a comprehensive description of the flow in a square-square contraction, in which the effects of both geometric and rheological parameters are analyzed, is still lacking.

In this paper, we move toward this goal by presenting detailed experimental results for the flow of Newtonian and Boger fluids through square-square contractions with several different contraction ratios. Besides reporting global characteristics such as flow patterns and vortex length, as in previous studies, we also present particle image velocimetry (PIV) measurements, and pressure drop measurements across the contraction. In addition we study the flow behavior in different planes of the geometry, in contrast with previous work in which only the center plane was analyzed $[19,20]$. Furthermore, we go beyond the previously studied $4: 1$ contraction and examine the effect of contraction ratio, defined as the ratio between the width of the large duct and the width of the smaller duct, on the flow characteristics for a wide range of Reynolds and Deborah numbers. The contraction ratio is known to have a major influence on the viscoelastic flow characteristics in converging configurations, as predicted for axisymmetric [6] and planar [5] contractions. Additionally, we present numerical results of 3D simulations for Newtonian fluids in the studied geometries.

In the following section the experimental set-up and methods are briefly described. Fluid characterization is discussed in Section 3. The numerical method and governing equations used in the numerical calculations are briefly outlined in Section 4. In Sections 5 and 6 we present and discuss in detail the experimental results for the Newtonian fluid and for the Boger fluid, respectively. Furthermore, the Newtonian results are compared with the numerical simulations. The final section of this paper summarizes the main conclusions of this work.

\section{Experimental set-up and methods}

\subsection{Experimental set-up}

Fig. 1 shows a diagram of the experimental set-up. The key part of the experimental rig is a square column of length $L=1.75 \mathrm{~m}$, which is composed of two parts: a fixed square duct, with width $2 \mathrm{H}_{1}=24.0 \mathrm{~mm}$, and an interchangeable duct of smaller crosssection which fits tightly inside the larger one. The inner width of the inset square channel can be set to $2 \mathrm{H}_{2}=10.0 \mathrm{~mm}, 6.0 \mathrm{~mm}$, $3.0 \mathrm{~mm}$ or $2.0 \mathrm{~mm}$, defining $2.4: 1,4: 1,8: 1$ and $12: 1$ contraction ratios, respectively. The upstream and downstream sections are denoted by subscripts 1 and 2, respectively. The dashed lines in Fig. 1 represent the air circulation lines (vacuum), which are used to promote the flow.

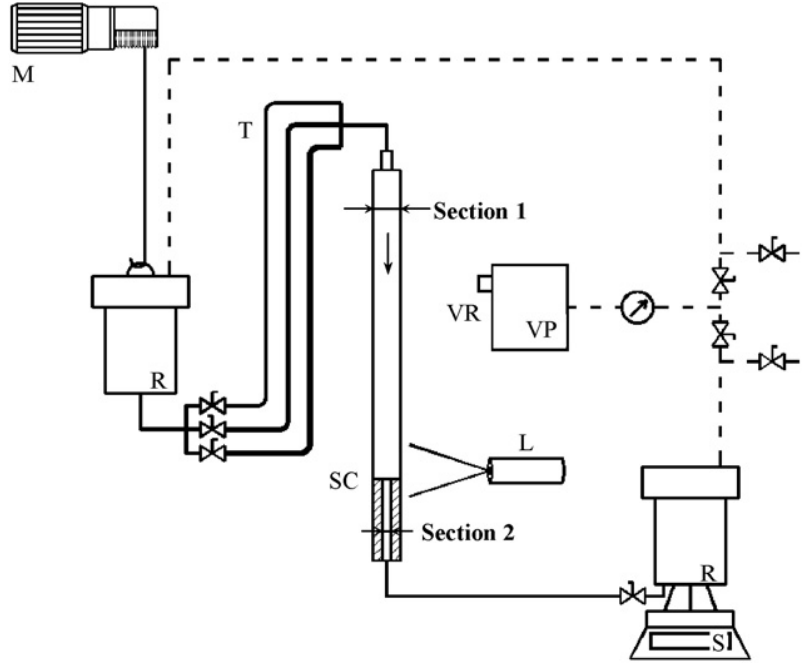

Fig. 1. Experimental set-up. (L, laser diode with cylindrical lens; M, electric motor with winch; R, reservoirs; S, weight scale; SC, square contraction; T, tubes; VP, vacuum pump; VR, vacuum regulator)

Table 1

Axial positions of the pressure ports $\left(\mathrm{P}_{0}, \mathrm{P}_{1}, \mathrm{P}_{2}\right.$ and $\left.\mathrm{P}_{3}\right)$. The contraction plane is located at $x=0$.

\begin{tabular}{lrrrr}
\hline & $C R=2.4$ & $C R=4$ & $C R=8$ & $C R=12$ \\
\hline$x_{0}(\mathrm{~mm})$ & -152 & -152 & -153 & -154 \\
$x_{1}(\mathrm{~mm})$ & +99 & +49 & +30 & +13 \\
$x_{2}(\mathrm{~mm})$ & +149 & +99 & +48 & +29 \\
$x_{3}(\mathrm{~mm})$ & +249 & +149 & +98 & +47 \\
\hline
\end{tabular}

The left hand-side reservoir (Fig. 1) is suspended in a steel cable connected to an electric motor, which allows the reservoir to be moved vertically by approximately $4 \mathrm{~m}$. This reservoir is connected to the upstream duct by three parallel tubes of different diameters, while the second reservoir is located on top of a weighing scale (KERN DS 16k0.2; with readout of $0.2 \mathrm{~g}$ and maximum range of $16 \mathrm{~kg}$ ) and is connected to the downstream duct by a $13 \mathrm{~mm}$ internal diameter tube.

The mass flow rate was set by adjusting the following parameters: the difference between the liquid levels in the reservoirs (attained by moving the vertical position of the left hand-side reservoir); the vacuum applied to the right hand-side reservoir using a vacuum pump (KNF Loboport N811-KT.18); and the diameter of the pipe that connects the reservoir to the duct (this diameter could be set to 4,6 or $15 \mathrm{~mm}$ ). The value of flow rate was monitored using LabView v7.1 that records the time and the mass of fluid in the right hand-side reservoir during the experiment. We note that we do not use any regulating valve to adjust the flow rate in order to avoid degradation of the fluid by passing through narrow orifices.

The experimental set-up was designed to provide a versatile mechanism that allows varying the contraction ratio, modifying the flow rate and adjusting the optical laser/camera arrangement in a simple and straight-forward way. For the purpose of flow visualization, the experimental set-up was made of transparent acrylic and the optical measurement system was adjusted to comply with the requirements of streak-line photography and PIV as described in

Table 2

Composition of fluids in mass concentrations and density.

\begin{tabular}{|c|c|c|c|c|c|c|}
\hline Fluid & PAA (ppm) & Glycerin (\%) & Water (\%) & Kathon (ppm) & $\mathrm{NaCl}(\%)$ & $\rho^{\mathrm{a}}\left(\mathrm{kg} / \mathrm{m}^{3}\right)$ \\
\hline Newtonian & - & 84.84 & 15.16 & 25 & - & 1221 \\
\hline Boger & 100 & 90.96 & 7.52 & 28 & 1.50 & 1249 \\
\hline
\end{tabular}

\footnotetext{
a The density was measured at $293.2 \mathrm{~K}$.
} 


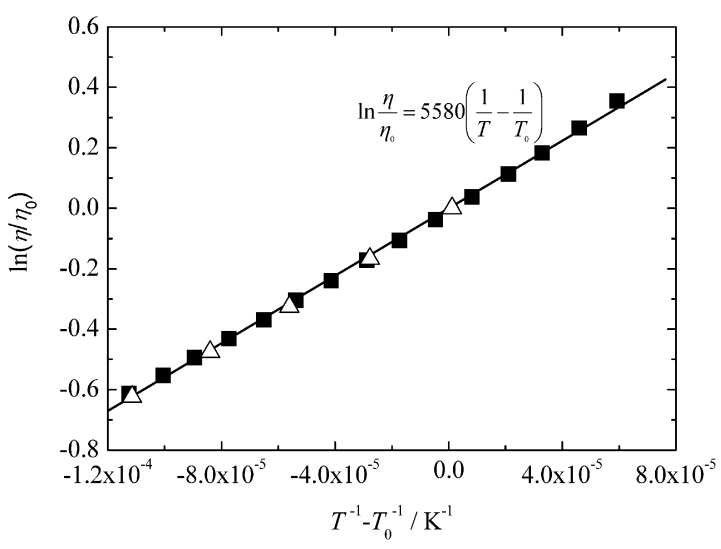

Fig. 2. Temperature effect on the shear viscosity for the Newtonian fluid studied ( $\mathbf{\square})$ and comparison with a $85 \mathrm{wt}$.\% glycerol solution $(\triangle)$, reported in Ref. [24] $\left(T_{0}=293.2 \mathrm{~K}\right)$.

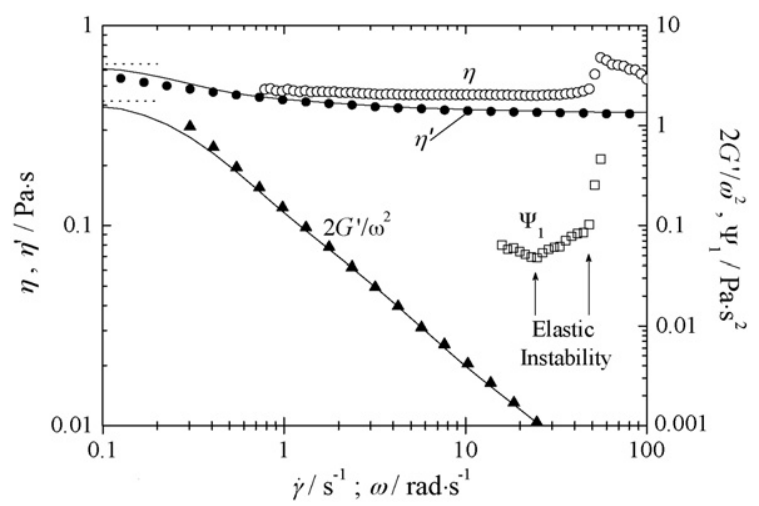

Fig. 3. Material functions for Boger fluid at reference temperature ( $293.2 \mathrm{~K})$ in steady (open symbols) and dynamic (solid symbols) shear flow.

Sections 2.2.1 and 2.2.2, respectively. To allow for pressure drop measurements (detailed in Section 2.2.3), four ports were positioned axially along the contraction geometry. One of the ports (the reference port, $\left.\mathrm{P}_{0}\right)$ was located upstream of the contraction plane $(x=0)$, and the remaining ports $\left(P_{1}, P_{2}\right.$ and $\left.P_{3}\right)$ were located downstream of the contraction at positions that depend on the size of the smaller duct. Table 1 displays the precise port locations for each contraction ratio considered.

\subsection{Experimental techniques}

\subsubsection{Streak-line photography}

A detailed study of the flow patterns was carried out using long time exposure streak-line photography. The fluid was seeded with highly reflective PVC tracer particles $10 \mu \mathrm{m}$ in diameter, at a concentration of approximately $30 \mathrm{ppm}$. The flow was continuously illuminated using a $635 \mathrm{~nm} 5 \mathrm{~mW}$ laser diode (Vector, model 520020) with a cylindrical lens incorporated in order to transform the spot laser beam into a light sheet that illuminates the plane under
Table 3

Linear viscoelastic spectra for the Boger fluid at the reference temperature $\left(T_{0}=293.2 \mathrm{~K}\right)$.

\begin{tabular}{lll}
\hline Mode $k$ & $\lambda_{k}(\mathrm{~s})$ & $\eta_{k}($ Pas $)$ \\
\hline 1 & 4.0 & 0.225 \\
2 & 0.4 & 0.04 \\
3 & 0.04 & 0.014 \\
Solvent & - & 0.367 \\
\hline
\end{tabular}

study. The streak-line images were captured using a digital camera (Canon EOS 30D) equipped with a macro lens (Canon EF100 mm, f/2.8) placed perpendicularly to the laser light sheet. The exposure time was varied depending on the flow conditions, ranging from $O(1 \mathrm{~s})$ up to $O\left(10^{3} \mathrm{~s}\right)$.

Most streak-line images were taken at the center plane, i.e. midway between the front and the back wall of the duct section. In some cases, streak-line images were also obtained at different parallel planes, which required simultaneous adjustment of the light source and the camera. This was accomplished using a manual 2D traverse and a dial comparator with a relative positioning precision of $\pm 0.01 \mathrm{~mm}$.

\subsubsection{PIV}

Particle Image Velocimetry was used to measure the velocity field in the vicinity of the contraction. The position of the optical components, such as the laser and the camera, is similar to that used for flow visualizations. However, PIV imaging requires very short exposure times compared with streak-line photography which typically uses exposure times on the order of seconds.

A doubled pulsed Nd:YAG laser, with a maximum energy of $50 \mathrm{~mJ}$ (Solo PIV III from New Wave Research), combined with appropriate optical components, produces a light sheet that illuminates the measurement plane. A digital CCD camera (Flow Sense 2M from Dantec Dynamics coupled with a Nikon AF Micro Nikkor $60 \mathrm{~mm}$ $\mathrm{f} / 2.8 \mathrm{D}$ lens), running on double frame mode and positioned perpendicularly to the light sheet, was used to acquire the images. The time interval between each frame was adjusted according to the flow rate, the interrogation area, and the section of the flow being analyzed.

The velocity field was determined by processing the images with FlowManager v4.60 software, using a cross-correlation algorithm to generate a two-dimensional velocity vector map for each image pair. Subsequently, the velocity field is submitted to a validation process using a moving average criterion. This process is carried out for a minimum of 60 double images for each steady-state experiment and an averaged velocity field is determined. In order to increase the accuracy of PIV measurements when the variation of velocity throughout the region of interest is high, the flow region was divided in smaller sub-regions and PIV measurements were performed independently for each subregion using an appropriate time between frames according to the local velocities. The full velocity field could then be reconstructed by compiling the data obtained for each sub-region. A thorough discussion of the PIV technique can be found in Refs. $[22,23]$.

Table 4

Characteristics of the two meshes used for each contraction ratio studied.

\begin{tabular}{|c|c|c|c|c|c|c|}
\hline & \multicolumn{2}{|l|}{$C R=2.4$} & \multicolumn{2}{|l|}{$C R=4$} & \multicolumn{2}{|l|}{$C R=8$} \\
\hline & M40 & M80 & M40 & M80 & M40 & M80 \\
\hline $\mathrm{NC}$ & 82,000 & 656,000 & 51,000 & 408,000 & 83,300 & 652,800 \\
\hline$\Delta x_{\min } /\left(2 H_{1}\right)$ & $2.08 \times 10^{-2}$ & $1.03 \times 10^{-2}$ & $1.31 \times 10^{-2}$ & $6.25 \times 10^{-3}$ & $7.50 \times 10^{-3}$ & $3.75 \times 10^{-3}$ \\
\hline$\Delta y_{\min } /\left(2 \mathrm{H}_{1}\right)=\Delta z_{\min } /\left(2 \mathrm{H}_{1}\right)$ & $1.99 \times 10^{-2}$ & $9.93 \times 10^{-3}$ & $1.25 \times 10^{-2}$ & $6.25 \times 10^{-3}$ & $7.50 \times 10^{-3}$ & $3.75 \times 10^{-3}$ \\
\hline
\end{tabular}



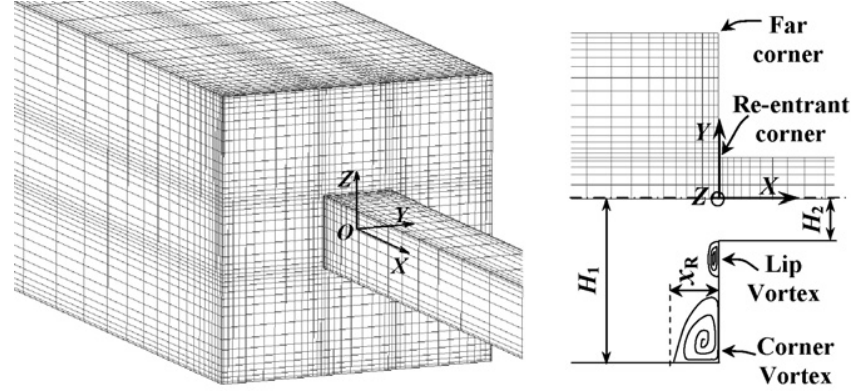

Fig. 4. Mesh M40 used in the numerical simulations of the flow through 4:1 contraction and identification of the coordinate system.

\subsubsection{Pressure drop measurement}

The pressure drop across the contraction region was measured using Honeywell 26PC differential pressure sensors (26PCA FA6D) which can cover a differential pressure range of $\pm 6.9 \mathrm{kPa}$. Each sensor is connected to the reference port $\mathrm{P}_{0}$, located upstream of the contraction plane, and one of the following ports located downstream of the contraction: $\mathrm{P}_{1}, \mathrm{P}_{2}$ or $\mathrm{P}_{3}$ (cf. Table 1 ). For the steady-state experiments, the transient output of the sensors was recorded until the steady value of pressure drop was reached. The

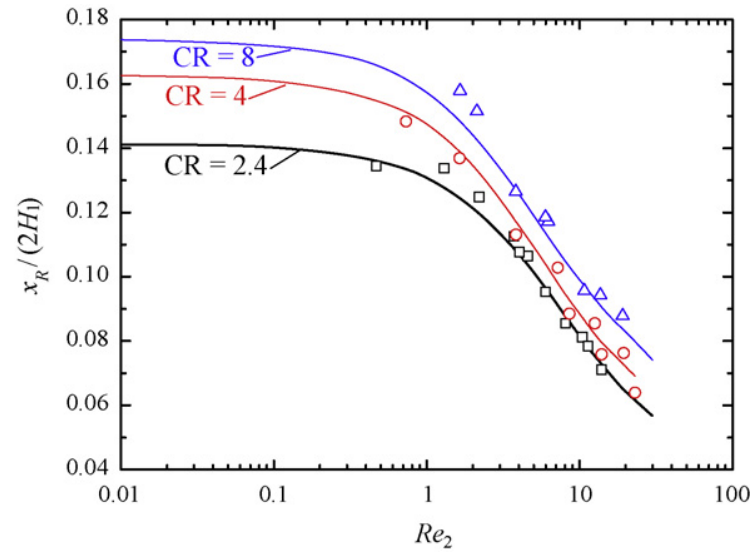

Fig. 6. Effect of the Reynolds number on the vortex length at the center plane of the square/square contraction for $C R=2.4(\square), C R=4(\bigcirc)$ and $C R=8(\triangle)$. Comparison with numerical calculations in mesh M80 (solid lines).

data was acquired through a data acquisition card (NI USB-6218, National Instruments) using LabView v7.1. Prior to use, the sensors were calibrated using a static column of water for the whole differential pressure range.
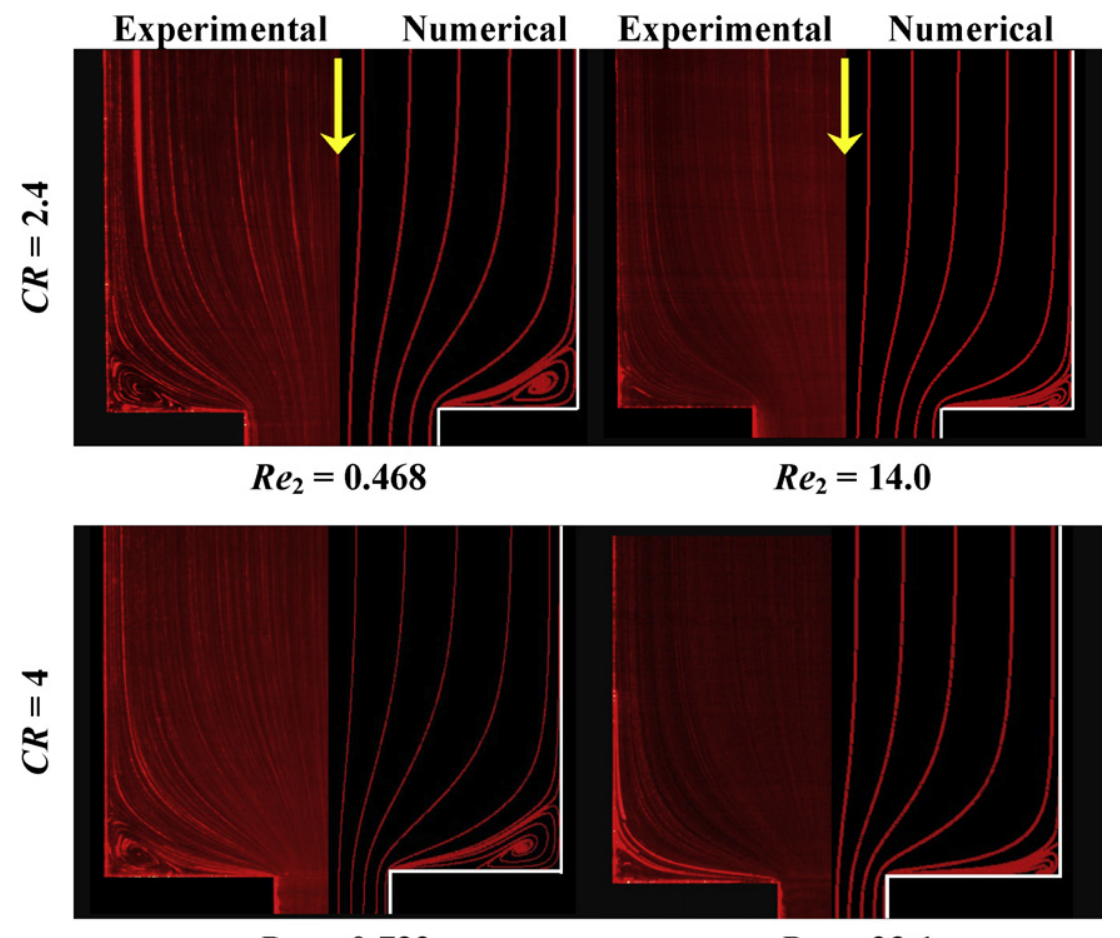

$R e_{2}=\mathbf{0 . 7 3 2}$

$R e_{2}=23.1$

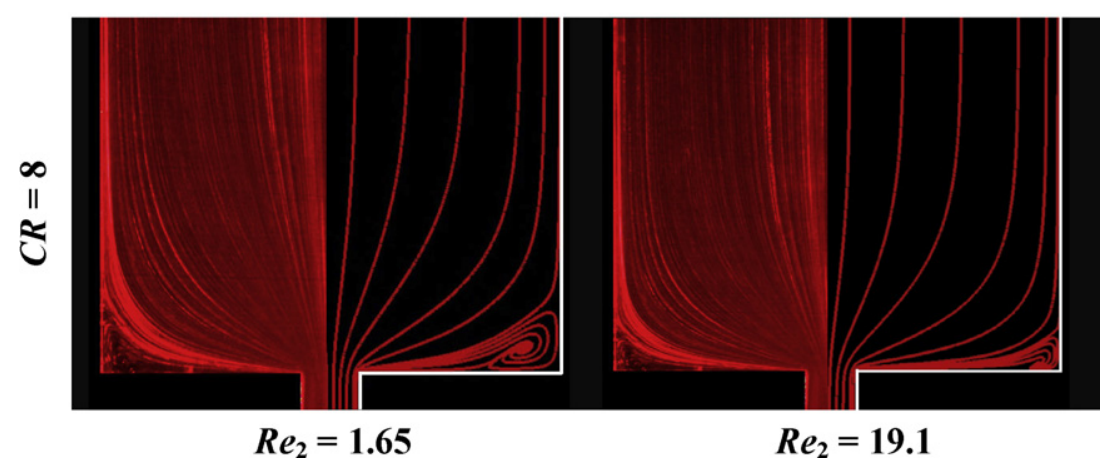

Fig. 5. Experimental and numerical streak lines at the center plane of the square/square contraction for different contraction ratios and Reynolds numbers. 


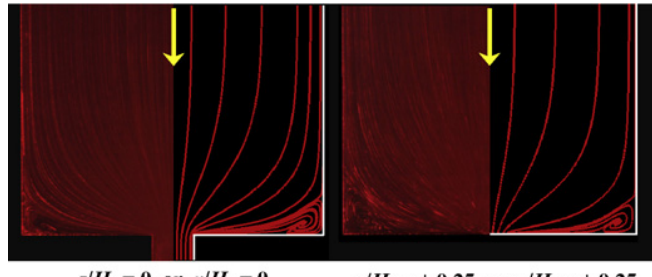

$z / H_{1}=0$ or $y / H_{1}=0$

$z / H_{1}= \pm 0.25$ or $y / H_{1}= \pm 0.25$

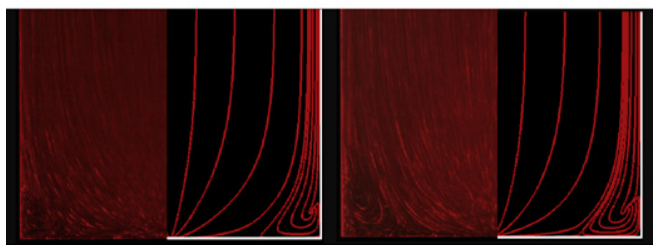

$z / H_{1}= \pm 0.50$ or $y / H_{1}= \pm 0.50 \quad z / H_{1}= \pm 0.75$ or $y / H_{1}= \pm 0.75$

Fig. 7. Experimental (left side) and numerical (right side) projected streak lines at different planes, for $R e_{2}=3.82$ of the $8: 1$ square/square contraction.

\section{Fluid characterization}

In the experiments we used either a Newtonian fluid or a Boger fluid. A summary of the composition of the fluids is given in Table 2 . The fluids were characterized using an MCR301 shear rheometer
(Anton Paar), and the density $(\rho)$ was measured using a hydrometer (readability of $0.001 \mathrm{~kg} / \mathrm{m}^{3}$; range $1200-1300 \mathrm{~kg} / \mathrm{m}^{3}$ ). Details of the rheological measurements are given in Sections 3.1 and 3.2 for the Newtonian and the Boger fluid, respectively.

\subsection{Newtonian fluid}

The Newtonian fluid used in this work was a solution of glycerol (84.8\%) and water (15.2\%). A biocide (Kathon LXE, Rohm and Haas) was added to the solution at a concentration of $25 \mathrm{ppm}$ in order to minimize bacteriological growth, and thus prevent fluid degradation.

The temperature effect on the steady shear viscosity was measured using the shear rheometer with a cone-plate fixture $(75 \mathrm{~mm}$ in diameter and $1^{\circ}$ angle), for temperatures ranging from $288.2 \mathrm{~K}$ to $303.2 \mathrm{~K}$. The results are shown in Fig. 2 for the Newtonian fluid and comparison is made against a $85 \%$ aqueous solution of glycerol as reported in Ref. [24].

The dependency of the shear viscosity on the temperature can be properly described by an Arrhenius equation in the form:

$\ln \left(a_{T}\right)=\frac{\Delta H}{R}\left(\frac{1}{T}-\frac{1}{T_{0}}\right)$

where $\Delta H$ represents the activation energy for flow, $T_{0}$ the reference absolute temperature ( $293.2 \mathrm{~K}), R$ the universal gas constant (a)

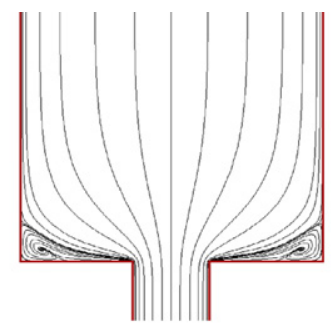

(b)

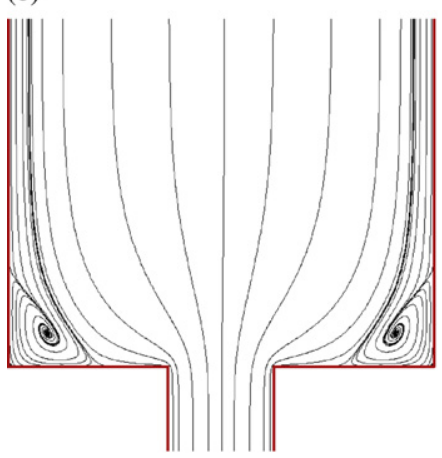

(c)

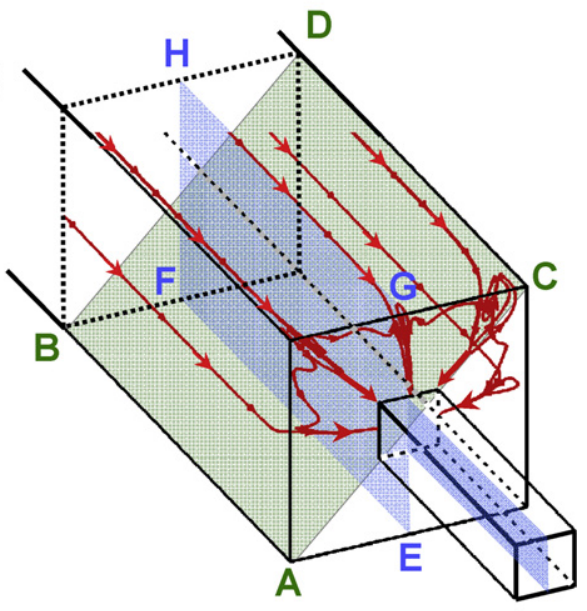

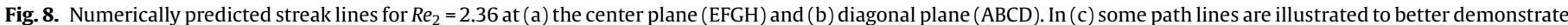
the three-dimensionality of the flow.
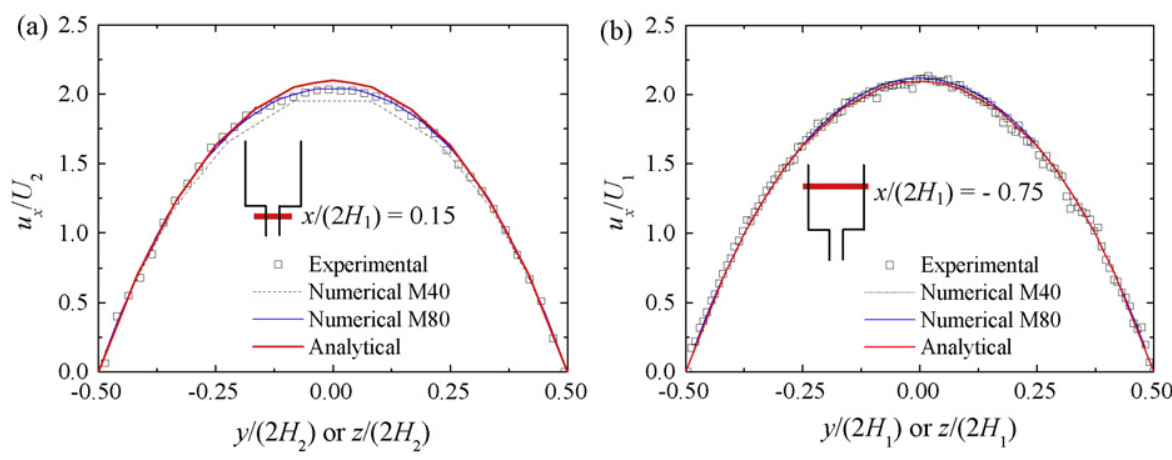

Fig. 9. Axial velocity profiles at the center plane for $C R=4$ and $R e_{2}=10.3$ at (a) $x /\left(2 H_{1}\right)=0.15$ in the downstream channel and (b) $x /\left(2 H_{1}\right)=-0.75$ in the upstream channel. 

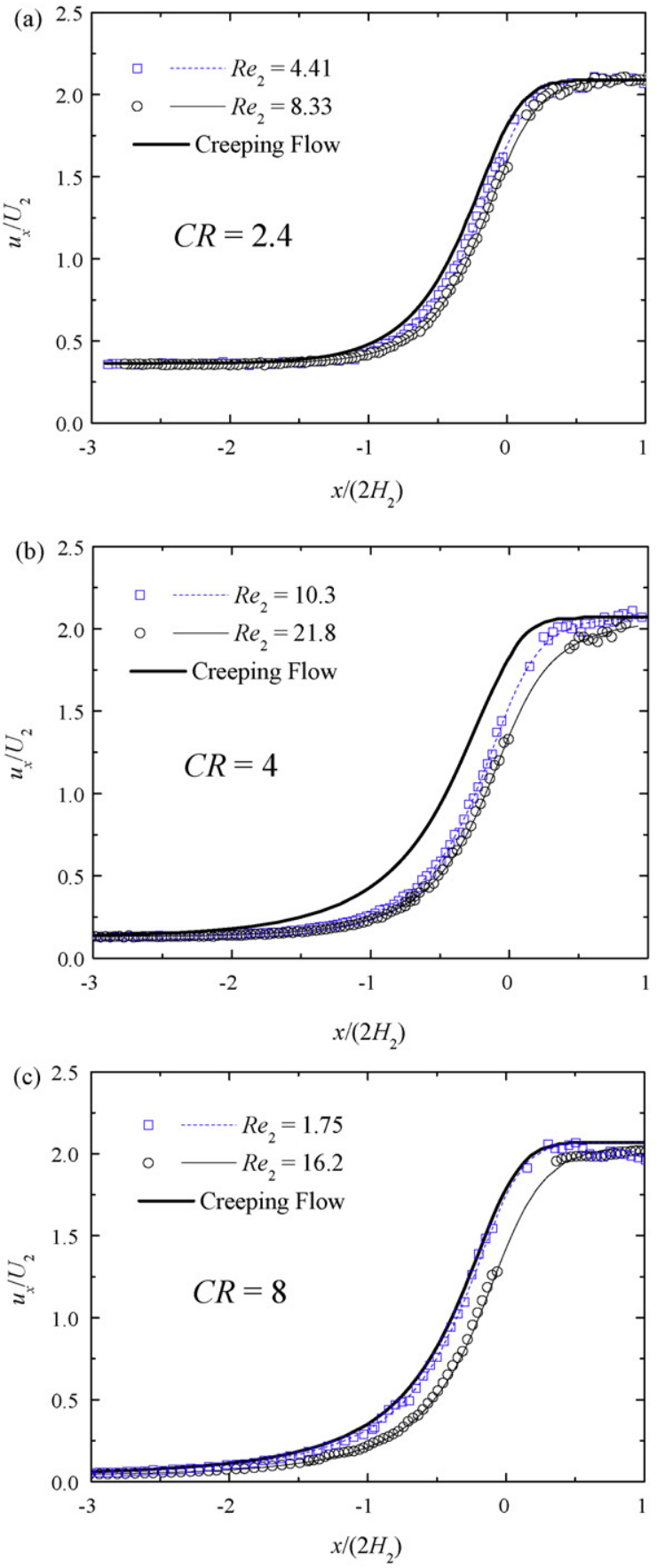

Fig. 10. Experimental axial velocity profiles (symbols) along the centerline at the center plane for (a) $C R=2.4$, (b) $C R=4$ and (c) $C R=8$ and comparison with numerical predictions (lines) in mesh M80.

and $a_{T}$ the shift factor, usually defined as [25]:

$a_{T}=\frac{\eta(T)}{\eta\left(T_{0}\right)} \frac{T_{0}}{T} \frac{\rho_{0}}{\rho}$

in which $\eta\left(T_{0}\right)$ is the shear viscosity at the reference temperature $T_{0}, \eta(T)$ is the shear viscosity at a given temperature $T$, while $\rho_{0}$ and $\rho$ are the fluid densities at the reference temperature and at temperature $T$, respectively. For the range of measurements, the temperature variation is limited and small enough to consider that the fluid density and the ratio $T_{0} / T$ do not change significantly. Thus,

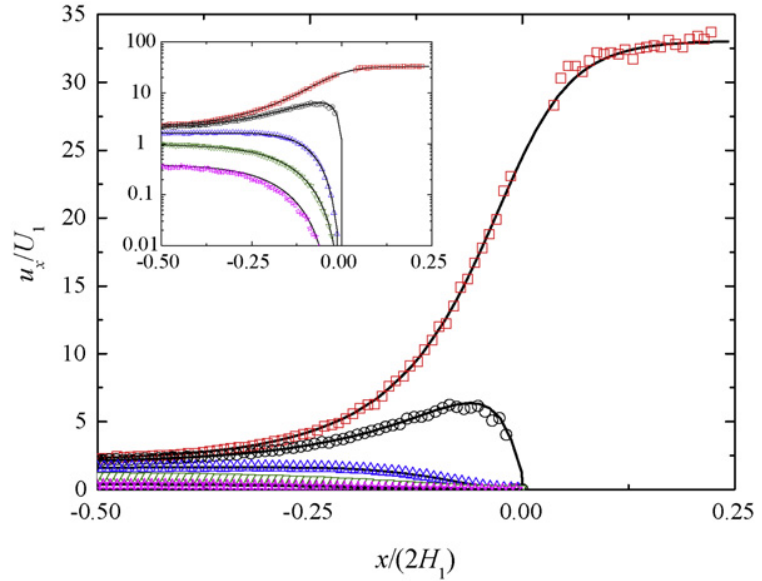

Fig. 11. Experimental (symbols) and numerical (lines) profiles of the axial velocity for $C R=4$ and $R e_{2}=10.3$ at $y=0$ and $z= \pm k H_{1}$ (or $z=0$ and $y= \pm k H_{1}$ ), where ( $\left.\square\right) k=0$; ( $\bigcirc)=0.25 ;(\triangle) k=0.50 ;(\nabla) k=0.75$; and $($ 数 $) k=0.92$.

the shift factor becomes [25]:

$a_{T}=\frac{\eta(T)}{\eta\left(T_{0}\right)}$

A fit of the experimental data to the Arrhenius equation (also shown in Fig. 2) demonstrates quantitatively the dependency of the shear viscosity with the temperature for the Newtonian fluid. The shear viscosity for the Newtonian fluid at the reference temperature is $\eta\left(T_{0}\right)=0.0982 \mathrm{~Pa}$ and $\Delta H / R=5580 \mathrm{~K}$.

\subsection{Boger fluid}

The Boger fluid was prepared by dissolving a small amount of PAA (100 ppm) into a Newtonian fluid of moderate viscosity. The shear viscosity $(\eta)$ and the first normal-stress difference coefficient $\left(\Psi_{1}\right)$ were measured in steady shear flow, and the storage and loss moduli ( $G^{\prime}$ and $G^{\prime \prime}$ ) in dynamic shear flow. A cone-plate geometry with $50 \mathrm{~mm}$ diameter and $2^{\circ}$ angle was used.

The steady shear tests were carried out for temperatures in the range between $283.2 \mathrm{~K}$ and $303.2 \mathrm{~K}$. The shift factors $a_{T}$ were determined and $\Delta H / R$ was calculated to be $6780 \mathrm{~K}$ using $293.2 \mathrm{~K}$ as the reference temperature. Fig. 3 displays the shear viscosity, $\eta$, as a function of the shear rate for the reference temperature, $T_{0}=293.2 \mathrm{~K}$. The shear viscosity of the Boger fluid is approximately constant for the whole range of shear rates tested $\left(0.7 \mathrm{~s}^{-1} \leq \dot{\gamma} \leq\right.$ $100 \mathrm{~s}^{-1}$ ). Also shown in this figure is the first normal-stress difference coefficient $\left(\Psi_{1}\right)$. For a critical shear rate between $20 \mathrm{~s}^{-1}$ and $50 \mathrm{~s}^{-1}$ an elastic instability sets in which originates a strong increase in the shear and normal stresses [26]. Above this critical shear rate the rheological measurements are not meaningful. We also show in Fig. 3 the dynamic shear data measured under small amplitude oscillatory shear (SAOS) flow, namely the dynamic shear viscosity $\left(\eta^{\prime}\right)$ and $2 G^{\prime} \mid \omega^{2}$ vs. $\omega$.

Since a single-mode model is not able to fit $G^{\prime}$ and $\eta^{\prime}$ accurately over the whole range of measured frequencies, instead a multimode model is used. The details of linear viscoelastic spectrum determined at $T_{0}=293.2 \mathrm{~K}$ are listed in Table 3 . The predictions using the three-mode model are overlapped with the experiments in Fig. 3 (solid lines) and it is clear that, within the measured frequency range, a three-mode model is adequate to represent the linear viscoelastic behavior of the fluid. The value of the solvent viscosity $\left(\eta_{\text {solvent }}=0.367 \mathrm{~Pa} \mathrm{~s}\right)$ of the three-mode model was chosen to match the shear viscosity of the N91 Newtonian fluid used by Alves et al. [19], which has a composition equivalent to the Boger fluid used here except, obviously, the N91 fluid contains no PAA. 


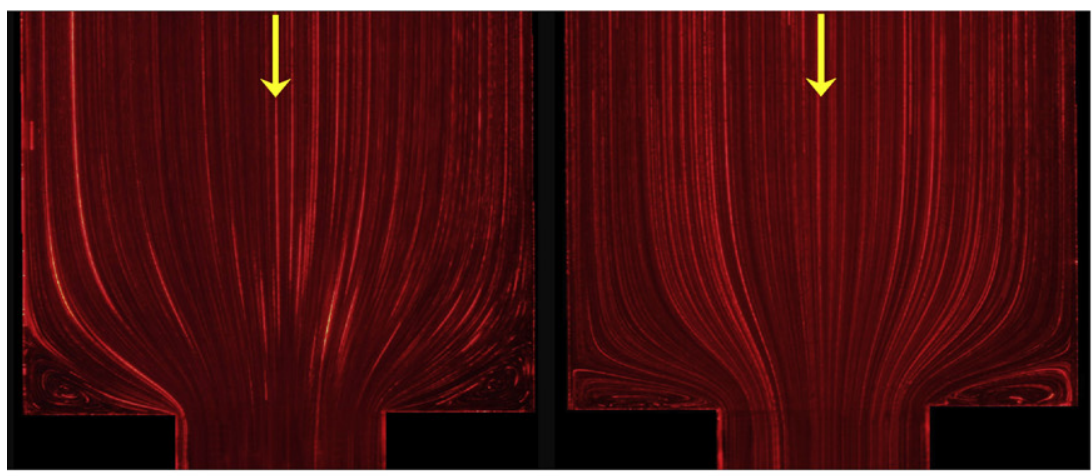

(a) $D e_{2}=8.55$

(b) $D e_{2}=25.68$

$\operatorname{Re}_{2}=\mathbf{0 . 4 7 2}$

$R e_{2}=1.11$

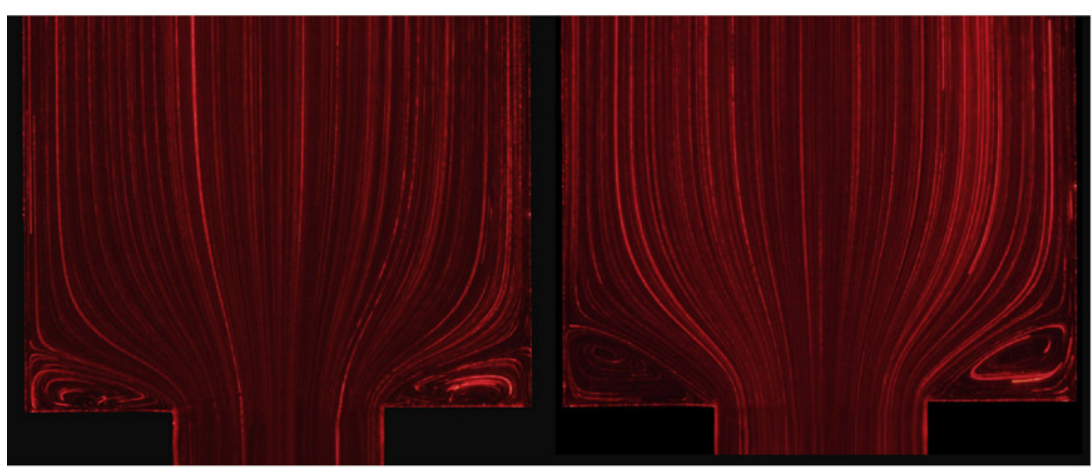

(c) $D e_{2}=29.3$

(d) $D e_{2}=42.9$

$R e_{2}=1.29$ $R e_{2}=1.88$

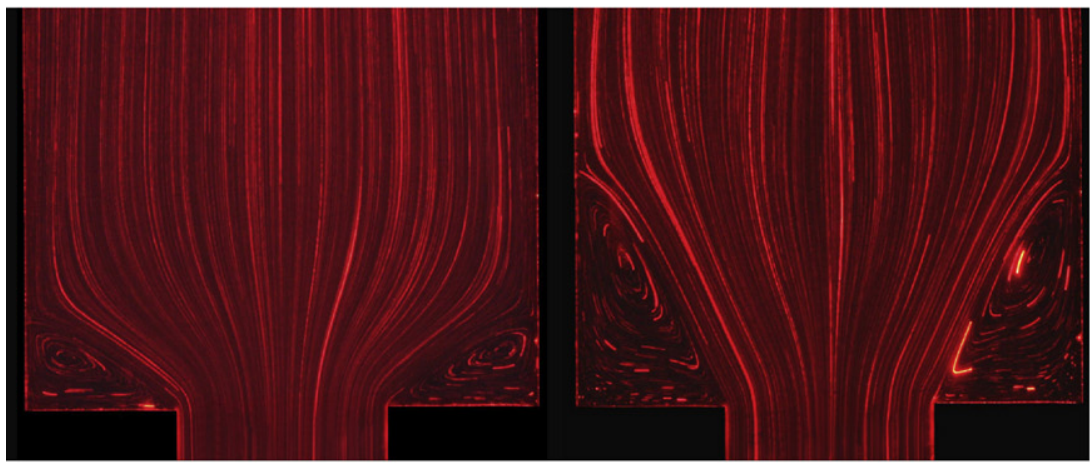

(e) $D e_{2}=64.5$

(f) $D e_{2}=92.3$

$R e_{2}=\mathbf{2 . 7 9}$

$R e_{2}=4.06$

Fig. 12. Effect of elasticity on the flow patterns at the center plane for $C R=2.4$.

Using the parameters of the three-mode model presented in Table 3, an average relaxation time can be calculated as:

$\lambda=\frac{1}{\eta_{p}} \sum_{k \neq \text { solvent }} \eta_{k} \lambda_{k}$

where $\eta_{p}=\sum_{k \neq \text { solvent }} \eta_{k}$, leading to $\lambda=3.29 \mathrm{~s}$. This average relaxation time will be used throughout this work in the determination of the Deborah number.

\section{Numerical method and governing equations}

The governing equations for steady, laminar flow of an incompressible fluid are those expressing the conservation of mass and momentum [25]:

$\nabla \cdot \mathbf{u}=0$ $\rho\left[\frac{\partial \mathbf{u}}{\partial t}+\mathbf{u} \cdot \nabla \mathbf{u}\right]=-\nabla p+\nabla \cdot \boldsymbol{\tau}$

where $\mathbf{u}$ is the velocity vector, $\rho$ the density of the fluid, $t$ the time, $p$ the pressure and $\tau$ the extra stress tensor, defined as the sum of a Newtonian solvent and a polymeric solute contribution $\left(\boldsymbol{\tau}=\boldsymbol{\tau}_{\mathbf{s}}+\boldsymbol{\tau}_{\mathbf{p}}\right)$.

For Newtonian fluids, $\boldsymbol{\tau}_{\mathbf{p}}=0$ and the Newtonian solvent component $\boldsymbol{\tau}_{\mathbf{s}}$ is expressed by:

$\boldsymbol{\tau}_{\mathbf{s}}=\eta_{\mathrm{s}}\left(\nabla \mathbf{u}+\nabla \mathbf{u}^{\mathrm{T}}\right)=2 \eta_{\mathrm{s}} \mathbf{D}$

where $\eta_{\mathrm{s}}$ is the constant solvent viscosity.

The set of governing equations, i.e. the mass and momentum conservation given by Eqs. (5) and (6), together with the constitutive equation for Newtonian fluids (Eq. (7)) are solved numerically using a fully-implicit finite volume method as explained in detail by Oliveira et al. [27]. 


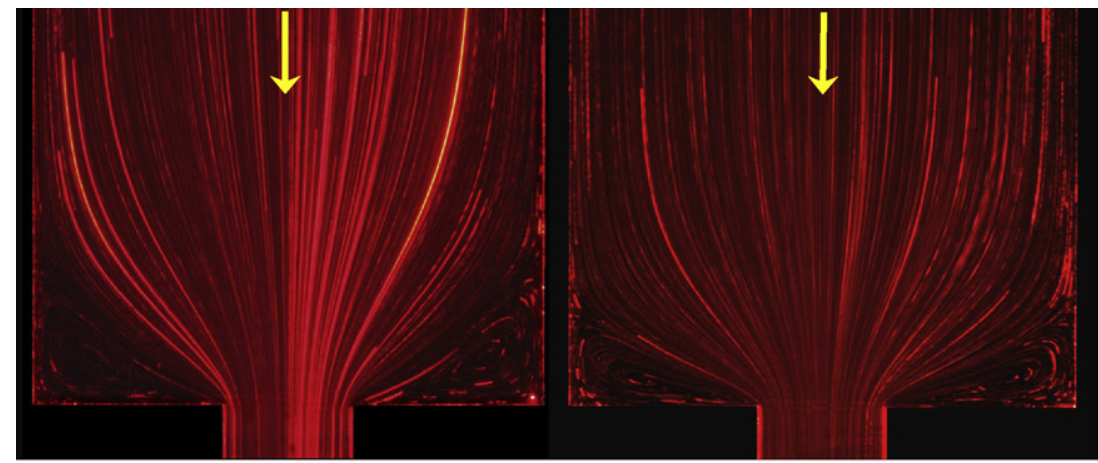

(a) $D e_{2}=3.00$

(b) $D e_{2}=10.3$

$R e_{2}=0.0514$

$R e_{2}=0.175$

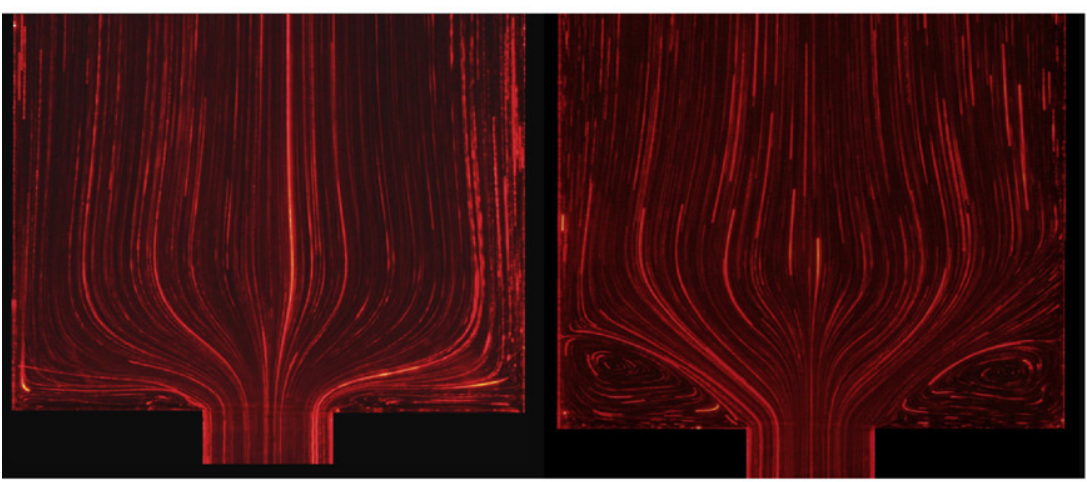

(c) $\mathrm{De}_{2}=33.6$

(d) $D e_{2}=47.5$

$R e_{2}=0.491$

$R e_{2}=0.878$

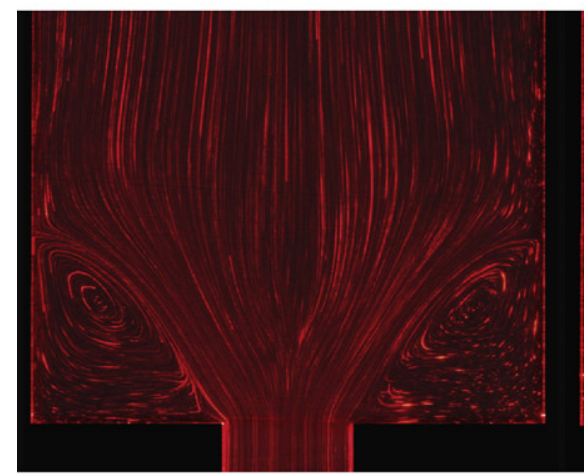

(e) $D e_{2}=\mathbf{5 5 . 3}$

$R e_{2}=0.902$

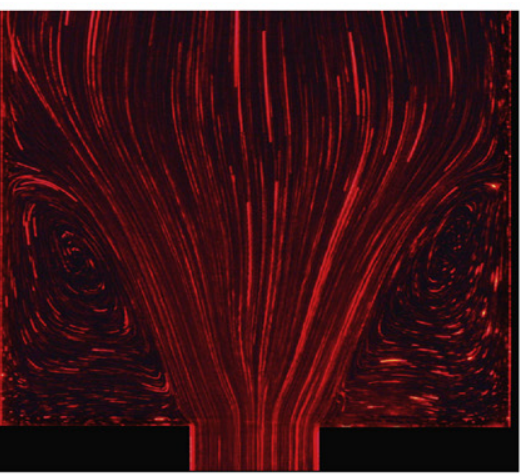

(f) $\mathrm{De}_{2}=62.7$

$R e_{2}=1.12$

Fig. 13. Effect of elasticity on the flow patterns at the center plane for $C R=4$.

The governing equations are discretized in time over a small time step $(\delta t)$ and in space by integration over control volumes (CV) used to divide the computational flow domain. The time derivative is discretized with an implicit first-order Euler scheme, while the diffusive terms are discretized with central differences. For accurate discretization of the advective terms we use the CUBISTA high-resolution scheme [28].

All numerical calculations were carried out using computational meshes representing the full wall-to-wall geometries. The overall dimensions of the square-square contractions are identical to those used experimentally (cf. Section 2.1), except the inlet and outlet planes that were located away from the region of interest (at $x /\left(2 H_{1}\right)=-25$ and $x /\left(2 H_{1}\right)=+25$, respectively), so that fullydeveloped flow conditions were enforced.

The computational domain was structured in orthogonal blocks composed by non-uniform cells. For each contraction ratio studied $(2.4: 1,4: 1$ and $8: 1)$, two meshes were used to map the square-square contraction in order to assess the accuracy of the numerical solutions. The coarse mesh (M40) has 40 cells in the largest duct along each of the two directions normal to the flow. The refined mesh (M80) has twice more cells in each of the three directions. Table 4 summarizes the characteristics of the meshes: the total number of cells (NC) and the dimensions of the smaller cell in the mesh, normalized by the width of the upstream duct, $2 \mathrm{H}_{1}$, for the range of contraction ratios studied.

Fig. 4 shows a three-dimensional representation of the coarse mesh (M40) used to simulate the 4:1 contraction ratio geometry. Clustering of cells near the re-entrant corners (cf. Fig. 4) was imposed in order to achieve higher accuracy in these regions, where the velocity and stress gradients are high. We have also included in Fig. 4 some important variables and notation used throughout the text. 


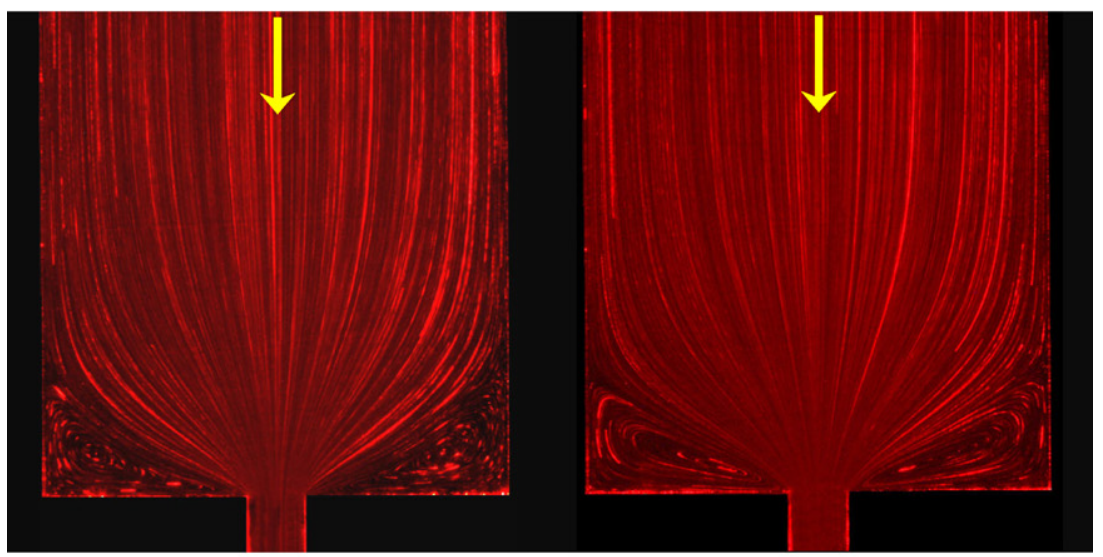

(a) $D e_{2}=6.00$

$R e_{2}=0.0273$ (b) $D e_{2}=12.4$

$R e_{2}=\mathbf{0 . 0 5 3 8}$

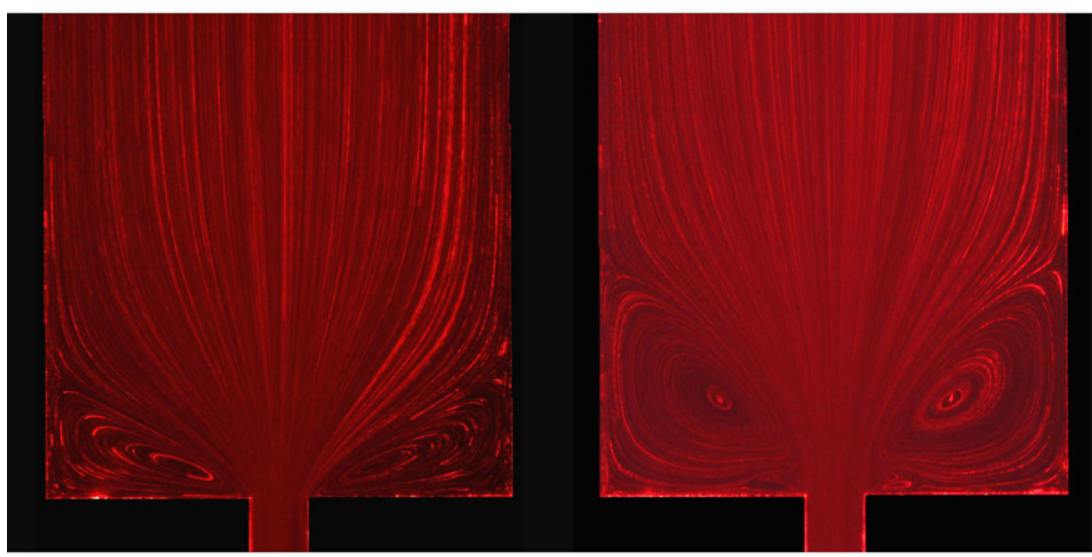

(c) $D e_{2}=18.1$

(d) $D e_{2}=47.5$

$R e_{2}=0.0824$

$R e_{2}=0.188$

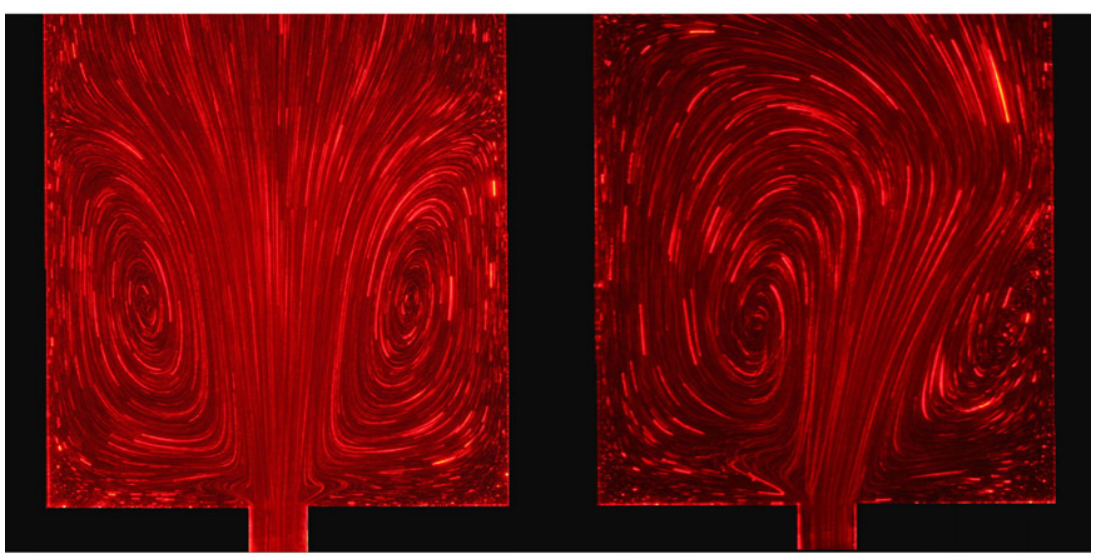

(e) $D e_{2}=89.7$

$R e_{2}=\mathbf{0 . 3 5 4}$

(f) $D e_{2}=116$

$R e_{2}=0.497$

Fig. 14. Effect of elasticity on the flow patterns at the center plane for $C R=8$.

\section{Newtonian fluid flow}

The study of the flow of a Newtonian fluid through 3D square-square contractions was carried out for three different contraction ratios: $C R=2.4,4$ and 8 . In this section we present the experimental and numerical results of flow patterns, vortex size and velocity field. The results were obtained experimentally using the Newtonian fluid and were simulated numerically using the computational meshes presented in Section 4.

\subsection{Flow patterns and vortex length}

The patterns generated by the Newtonian fluid flowing through the square-square contractions are presented in Fig. 5. For each $C R$, the streak lines obtained experimentally and the correspond- 


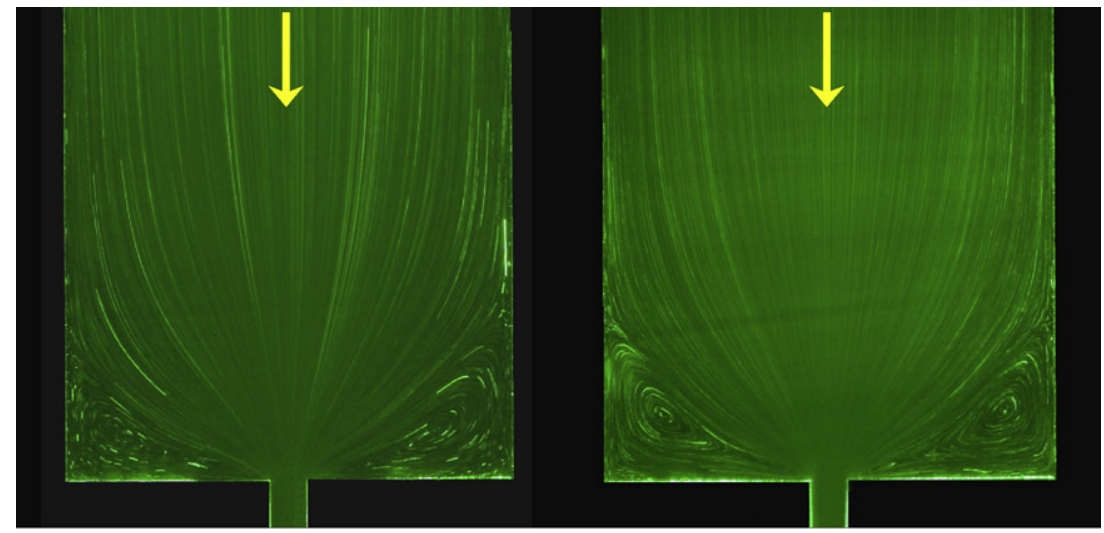

(a) $D e_{2}=8.03$ $R e_{2}=0.0148$ (b) $D e_{2}=9.95$ $R e_{2}=0.0178$

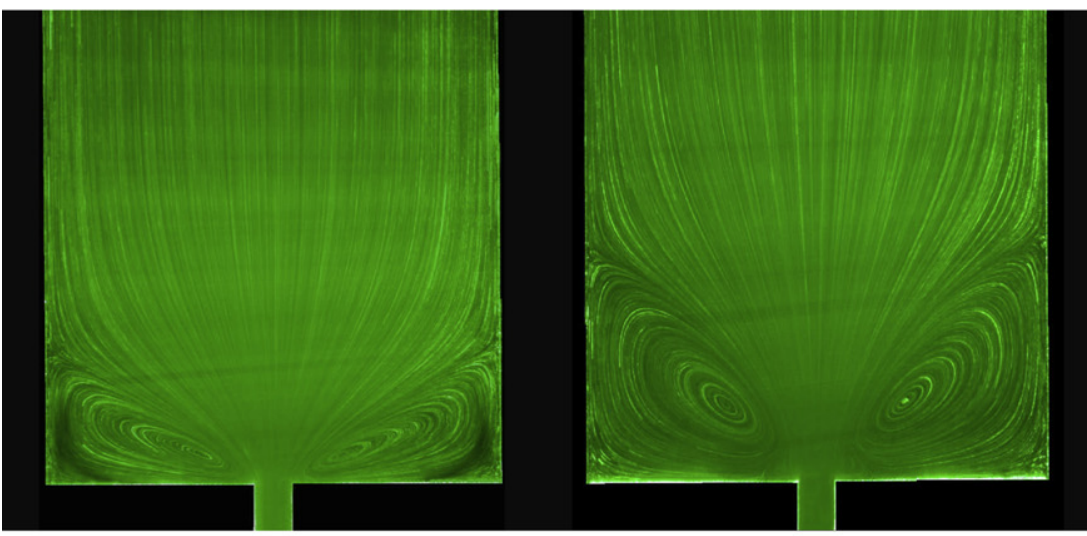

(c) $D e_{2}=26.4$ $R e_{2}=\mathbf{0 . 0 5 2 5}$ (d) $D e_{2}=37.5$ $R e_{2}=0.0769$

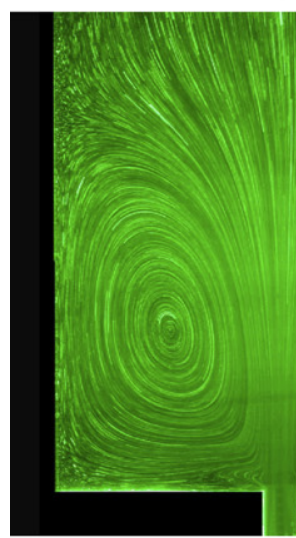

(e) $D e_{2}=88.6$ $R e_{2}=0.163$ (f) $D e_{2}=138$

$R e_{2}=\mathbf{0 . 2 5 5}$

Fig. 15. Effect of elasticity on the flow patterns at the center plane for $C R=12$.

ing numerical predictions are shown at the center plane $(y=0$ or $z=0$ ) for two different Reynolds numbers, here defined in terms of downstream variables:

$R e_{2}=\frac{\rho U_{2}\left(2 H_{2}\right)}{\eta}$

For the range of contraction ratios and $\mathrm{Re}_{2}$ studied, we observe the formation of a vortical structure upstream of the contraction near the far corner, i.e. corner vortex (cf. schematics in Fig. 4), which is in agreement with previous studies in square-square contractions $[14,15,19]$.
In Fig. 6 we show the variation of the vortex length, $x_{R}$, defined as the distance between the contraction plane and the top of the recirculation (cf. sketch in Fig. 4), with the Reynolds number for the three contraction ratios studied. The vortex length was measured at the center plane and its value was normalized using the width of the upstream duct, $2 \mathrm{H}_{1}$. Note that the characteristic dimension used in the Reynolds number is the width of the downstream channel, $2 \mathrm{H}_{2}$, which is different for each contraction ratio. We see that, for all $C R$, the experimental results are well predicted by the numerical simulations (cf. Fig. 6). 

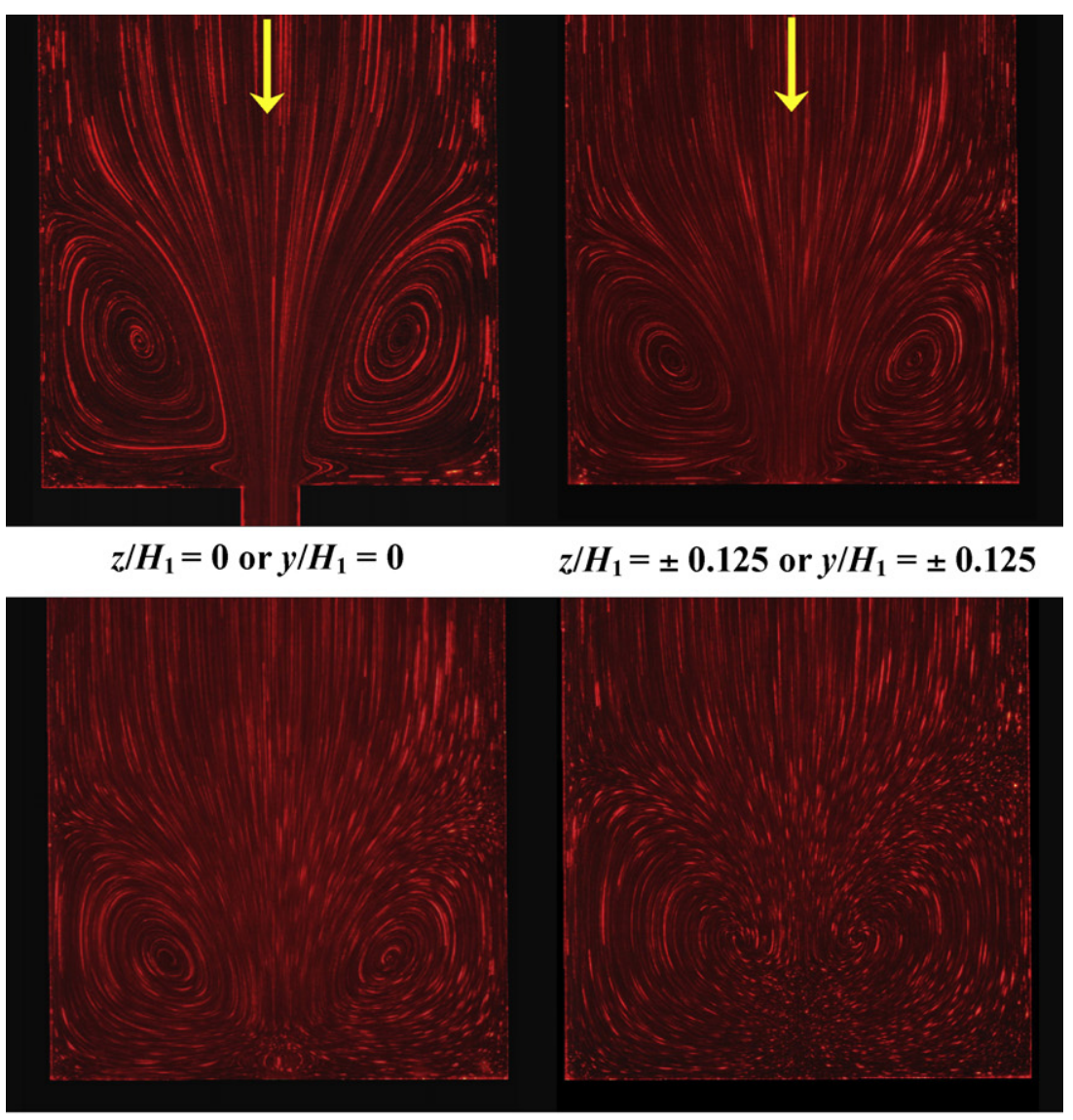

$$
z / H_{1}= \pm 0.25 \text { or } y / H_{1}= \pm 0.25 \quad z / H_{1}= \pm 0.50 \text { or } y / H_{1}= \pm 0.50
$$

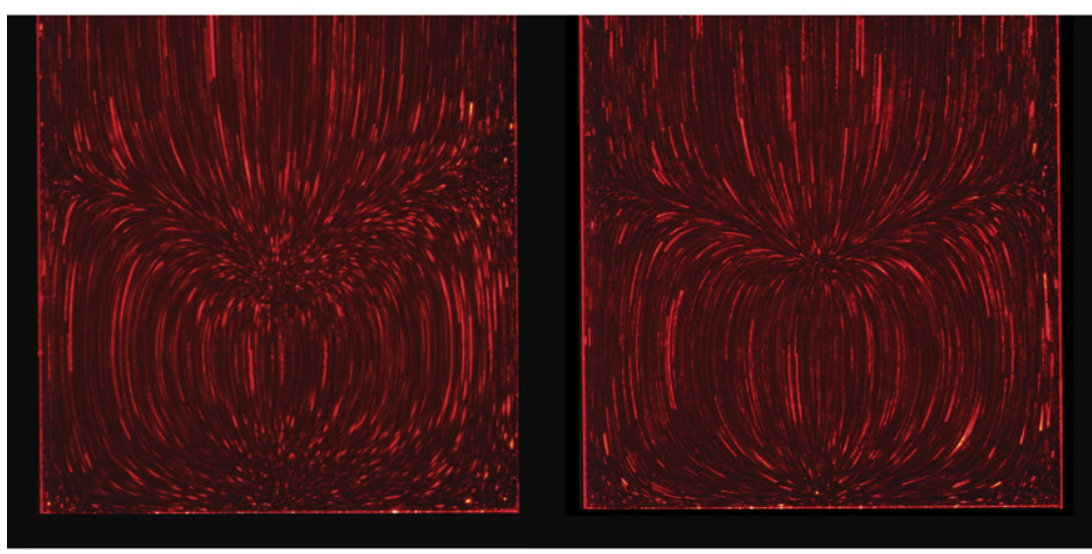

$$
z / H_{1}= \pm 0.75 \text { or } y / H_{1}= \pm 0.75 \quad z / H_{1}= \pm 0.917 \text { or } y / H_{1}= \pm 0.917
$$

Fig. 16. Streak line images at different planes of the square-square contraction for $D e_{2}=67.9, \operatorname{Re}_{2}=0.264$ and $C R=8$.

For low Reynolds numbers $\left(R e_{2} \leq 0.5\right)$, the dimensionless vortex length, $x_{\mathrm{R}} /\left(2 \mathrm{H}_{1}\right)$, determined numerically asymptotes to a plateau which is slightly dependent on the $C R: x_{R} /\left(2 H_{1}\right)=0.142$ for $C R=2.4 ; x_{R} /\left(2 H_{1}\right)=0.163$ for $C R=4$ (in agreement with Ref. [19]); $x_{R} /\left(2 H_{1}\right)=0.174$ for $C R=8$. The dependence of the plateau value of the vortex length on the contraction ratio was found to be nonlinear, analogously with the findings of Alves et al. [5] and Oliveira et al. [6] for planar and axisymmetric contractions, respectively. However, under creeping flow conditions the value of $x_{R} /\left(2 H_{1}\right)$ will become independent of $C R$ for very high contraction ratios. For higher Reynolds number $\left(\operatorname{Re}_{2} \geq 0.5\right)$, the length of the vortex decreases with increasing inertia for the three contraction ratios investigated.
Even though the flow inside the corner vortex looks bidimensional, in reality it exhibits a complex behavior, showing a 3D open vortical structure upstream of the contraction. This type of open 3D recirculation has been observed previously for a square-square contraction with $C R=4$ [19] and for 3D planar expansions $[11,29]$. To better illustrate the flow inside the vortical structure, streak-line images were taken at different planes. Fig. 7 shows the experimental and numerical streak-line projections in four different planes at several locations between the center plane and the wall plane for $C R=8$. Due to the three-dimensionality of the flow within the contraction, the streak-line images obtained experimentally, with the exception of that at center plane, correspond to the projected path lines of the PVC particles on the 


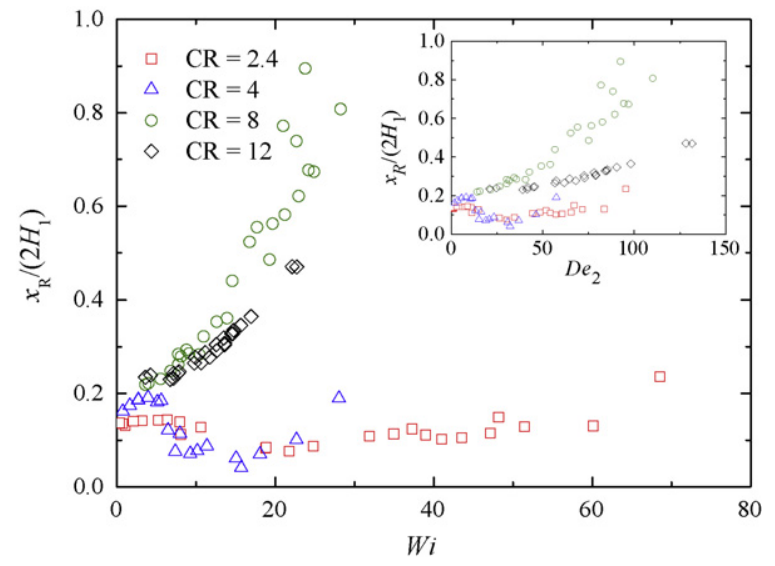

Fig. 17. Normalized vortex length as function of Wi for all contraction ratios studied. In the inset, the normalized vortex length is presented as a function of Deborah number.

illuminated plane. Additionally, in Fig. 8 we compare the streak lines at the center plane and at the diagonal plane predicted numerically.

The streak-line projections in a number of planes demonstrate that the particles in the upstream square duct enter the flow recirculation at the diagonal plane and exit at the center plane close to the re-entrant corner where they proceed into the downstream duct. The structure of these recirculations is open and threedimensional, similar to that observed experimentally and predicted numerically for Newtonian fluid flows through 3D sudden expansions $[11,29,30]$. Furthermore, the flow is symmetric relative to the two center planes $(y=0$ and $z=0)$ and to the two diagonal planes $(y= \pm z)$ due to the symmetry of the geometry. Fig. 8c (adapted from Ref. [19]) shows an overview of the 3D particle trajectories of a Newtonian fluid under low Reynolds number flow conditions. Under steady-state flow conditions, the recirculating vortex structures develop and the particles flowing close to the wall along the diagonal plane $(A B C D)$ enter the corner vortex, rotate toward its center, and then drift to the center plane (EFGH). After rotating toward the vortex periphery at the center plane, the particles exit toward the downstream duct, near the re-entrant corner as described in detail in Ref. [19].

\subsection{Velocity field}

In Fig. 9, we show the axial velocity profiles along the spanwise direction measured downstream and upstream of the $4: 1$ contraction. The position represented in the abscissa is normalized using the width of the corresponding duct and the axial velocity was scaled with the relevant average velocity. For clarity, we also show as inset a diagram of the location where the profiles were taken.

In the downstream channel close to the centerline $\left(y /\left(2 \mathrm{H}_{1}\right)=0\right.$ in Fig. 9a), where the maximum velocity is reached, the coarse mesh (M40) describes the experimental results with a maximum relative error of approximately $5 \%$, but the agreement is significantly improved by using the refined mesh (M80). In this case, the relative error between the numerical and experimental values becomes negligible, within the experimental error. In the upstream channel (Fig. 9b), the average velocity is 16 times smaller than that in the downstream channel. In this case, the two meshes used in the numerical calculations provide similar results, and the experimental velocity profile is well captured. Since the maximum relative error incurred when using the coarse mesh is small, mesh M40 might be useful when simulation time is a constraint, because the CPU time is substantially smaller than when using the M80 mesh. Note that the M80 mesh has eight times more cells than mesh
M40 and therefore CPU times are about one order of magnitude higher. The small differences observed between the experimental (or numerical) results and the analytical solution in Fig. 9a are due to the fact that at that axial location the fully-developed velocity profile is not yet observed since it is too close to the entrance of the contraction. This is evident in Fig. 10b, where we can observe that at $x /\left(2 H_{1}\right)=0.15\left(x /\left(2 H_{2}\right)=0.60\right)$ the centerline velocity is still below the fully-developed value.

Fig. 10 depicts the effect of inertia on the axial velocity profile along the centerline ( $y=0$ and $z=0$ ) for three values of $C R$. These experimental profiles display some scatter in the vicinity of the contraction plane, due to a shade that forms at the contraction plane when the laser light sheet illuminates the channel, which reduces the visibility of tracer particles, therefore leading to a reduction of the precision of the PIV results in this region of the flow. Nevertheless, we can see that the fluid accelerates as it approaches the contraction and an increase in $R e_{2}$ leads to a delay in the increase of the dimensionless velocity along the centerline and it takes longer for the flow to fully develop downstream of the contraction plane. The agreement between the experimental and numerical results is again very good for all $C R$.

The axial velocity profile along the streamwise direction for various lines $y=0$ and $z=k H_{1}$ (or identically for $z=0$ and $y=k H_{1}$ ) where $k=0,0.25,0.50,0.75$ and 0.92 , is shown in Fig. 11. As expected, the magnitude of the axial velocity component increases as we move from the wall to the center plane. The three profiles closer to the wall show a velocity decrease along the streamwise direction as the fluid approaches the contraction region due to the presence of the contraction plane wall at $x=0$. At the center plane, the fluid accelerates towards the contraction and the maximum strain rate is attained. For the plane $z / H_{1}=0.25$, which is coincident with the downstream channel wall, the profile displays an interesting behavior: there is a significant acceleration toward the contraction, followed by an abrupt decrease as we move towards the wall at the contraction plane. The velocity profiles obtained at planes away from the center plane and in particular at the plane $z / H_{1}=0.25$ reveal the flow structure in the recirculation zones. Again good quantitative agreement between experiments and numerical simulations was found, thus demonstrating the good accuracy of the experimental technique, which will also be applied to the flow of Boger fluids in the next section.

\section{Boger fluid flow}

The study of the flow of a Boger fluid through 3D square-square contractions was carried out for four different contraction ratios, $C R=2.4,4,8$ and 12 . The effect of Deborah number and contraction ratio on the flow patterns is examined in detail and, when appropriate, the corresponding vortex size is quantified. Furthermore, the velocity fields are measured at the center plane and at additional parallel planes closer to the wall. In this section we also present the experimental results of pressure drop across the contraction.

\subsection{Flow patterns and velocity field}

The effect of Deborah number on the flow patterns of the Boger fluid are illustrated in Figs. 12-15 for a range of contraction ratios. The photographs were taken at the center plane using the streak-line photography technique described in Section 2.2.1. To quantify the elastic effects we define the Deborah number based on downstream flow conditions and on the average relaxation time obtained by shear rheology as quantified in Section $3.2(\lambda=3.29 \mathrm{~s})$, $D e_{2}=\lambda U_{2} / H_{2}$. Experimentally, $D e_{2}$ was changed by varying the 
(a)

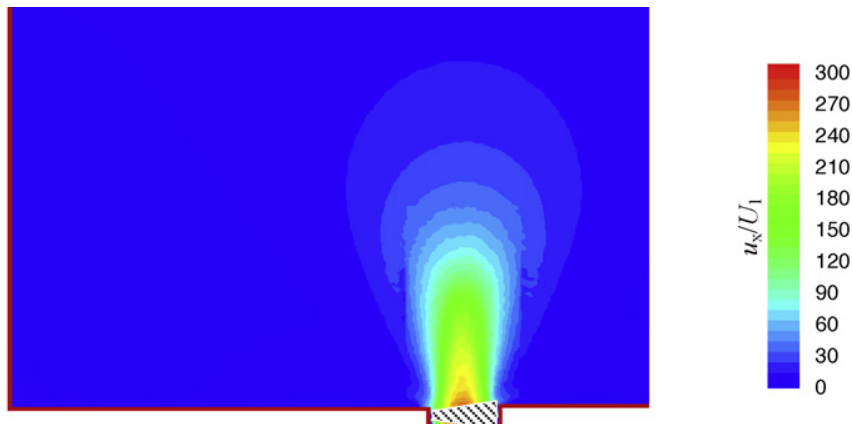

(b)

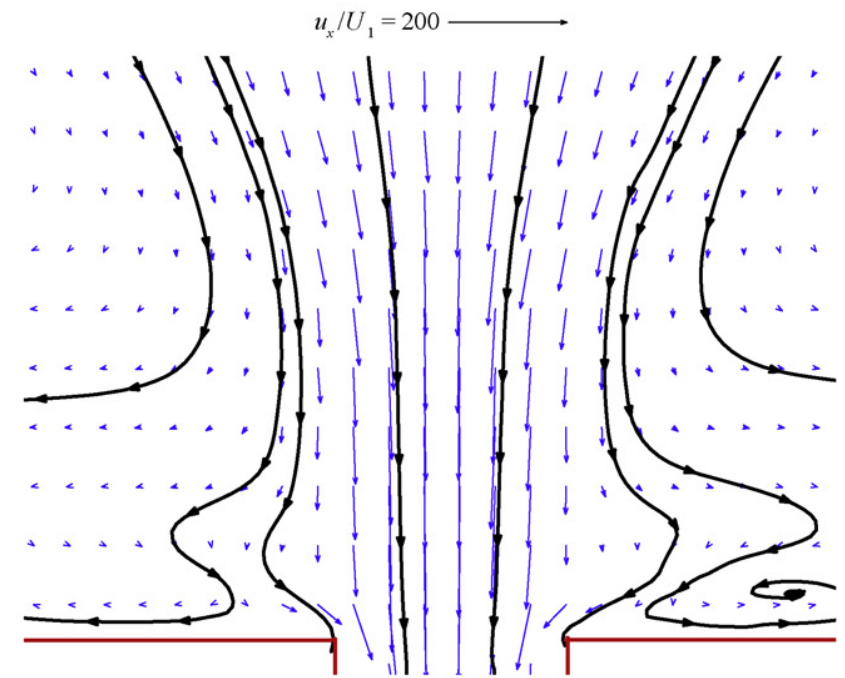

(c)

(d)

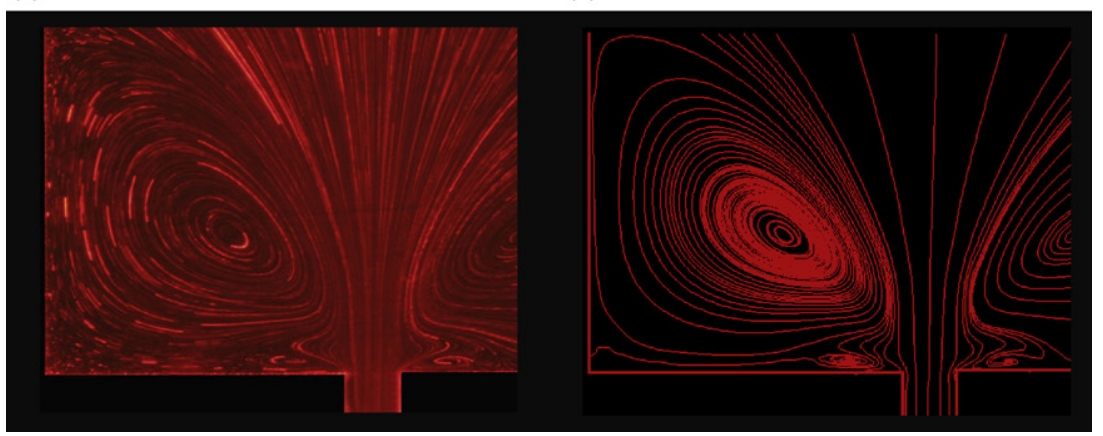

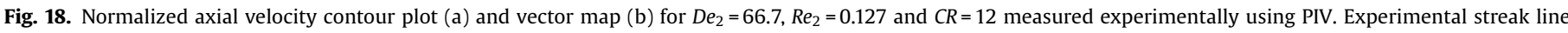
image (c) and streamlines obtained by integration of the velocity field (d) for the same flow conditions.

flow rate in the range $1.02 \times 10^{-8} \mathrm{~m}^{3} / \mathrm{s} \leq Q \leq 1.43 \times 10^{-5} \mathrm{~m}^{3} / \mathrm{s}$. Overall, fluid elasticity enhances the formation of complex structures upstream of the contraction and as the flow rate increases, a number of different flow type regions can be identified, such as: Newtonianlike flow; lip vortex activity, vortex growth and diverging flow; and unstable flow.

At low Deborah number flows, viscous effects predominate and Newtonian-like patterns are observed, with concaveshaped corner vortices forming upstream of the contraction (Figs. 12a, 13a, 14a and 15a). Initially, as $D e_{2}$ increases, the vortex shape and size do not seem to change significantly but as $D e_{2}$ is increased further, elastic effects become significant. In addition to the corner vortex a weak lip vortex (cf. sketch in Fig. 4) develops for $C R=4,8$ and 12 at a Deborah number $\left(D e_{2} \sim 10\right)$ which is approximately independent of the contraction ratio (Figs. 13b, 14b and 15b). The formation of lip vortices close to the re-entrant corner has also been discussed in Refs. [14,15,19] for flows of Boger fluids in 4:1 square-square contractions.

At high flow rates (Deborah numbers), elastic effects are enhanced and eventually lead to a change of the flow direction in the recirculations, i.e. the direction of fluid motion inside the vortices is the opposite of what is seen for lower flow rates (i.e. lower Deborah numbers) and for Newtonian fluids. This is observed for $D e_{2} \gtrsim 35,15,25$ and 30 for $C R=2.4,4,8$ and 12 , respectively. In Fig. 16, we show the streak-line images obtained at different planes by varying the distance from the center plane to the wall plane for $C R=8$ and $D e_{2}=67.9$. We can see that the tracer particles enter the corner vortex through the center plane contrary to the observations with the Newtonian fluid in which the particles enter the recirculation through the diagonal plane and exit through the central plane, as shown in Fig. 8. The reversal of the flow pattern was not reported for the Boger fluid studied in Ref. [19] but was 
observed and predicted numerically for shear-thinning fluids [20]. Therefore, we conclude that shear-thinning behavior is not necessary to produce reversal of the flow in the recirculation and that the flow inversion is due to strong elastic effects and in particular due to the high values of the extensional viscosity, as discussed in Ref. [20].

The size of the corner-vortex structure is found to depend non-monotonically on the Deborah number, in resemblance with previous observations in 4:1 axisymmetric contractions [26] and 4:1 square-square contractions [19]. Since in the region just upstream of the contraction the flow is highly extensional, we can define a Weissenberg number based on the relaxation time and on the estimated strain rate along the centerline, $W i=\lambda \dot{\varepsilon} \cong$ $\lambda\left(U_{2, \mathrm{c}}-U_{1, \mathrm{c}}\right) / H_{1} \cong 2.1\left[\left(1-1 / C R^{2}\right) / C R\right] \lambda U_{2} / H_{2}$ (note that for a square channel the centerline velocity of a fluid with constant shear viscosity is nearly 2.1 times the average velocity in the channel: $U_{2, \mathrm{c}} / U_{2}=U_{1, \mathrm{c}} / U_{1} \approx 2.1$ ), where $U_{1, \mathrm{c}}$ and $U_{2, \mathrm{c}}$ are the centerline velocities on the upstream and downstream channels, respectively. For square channels we can easily convert the Deborah number defined earlier in this section into a Weissenberg number: $W i=$ $2.1\left[\left(1-1 / C R^{2}\right) / C R\right] D e_{2}$. The variation of the dimensionless vortex length (normalized with the upstream channel width, $2 \mathrm{H}_{1}$ ) as function of $W i$ is shown in Fig. 17 for the range of contraction ratios tested. The vortex features observed in low $(C R=2.4$ and 4$)$ and high ( $C R=8$ and 12) contraction ratio geometries are distinct. The curves for high contraction ratios overlap when plotted as a function of $W i$ ( or $D e_{2} / C R$ ), in accordance with the scaling results of Alves et al. [5] and Oliveira et al. [6] for planar and axisymmetric geometries, respectively. In these cases, $D e_{2}$ enhances vortex growth and the flow features in the far corner area are only affected by upstream events [6]. Thus, the behavior for different $C R$ is similar and the curves coincide for high $C R$. For the low contraction ratios, the vortex growth regime is preceded by a decrease in vortex size until a minimum size is reached for $D e_{2} \sim 30$ (cf. inset in Fig. 17). As this reduction takes place, the shape of the vortex changes and becomes "convex" (cf. Figs. 12c, 12d and 13e).

In the vortex growth regime, the flow behavior in low and high contraction ratio geometries is distinct as pointed out previously.

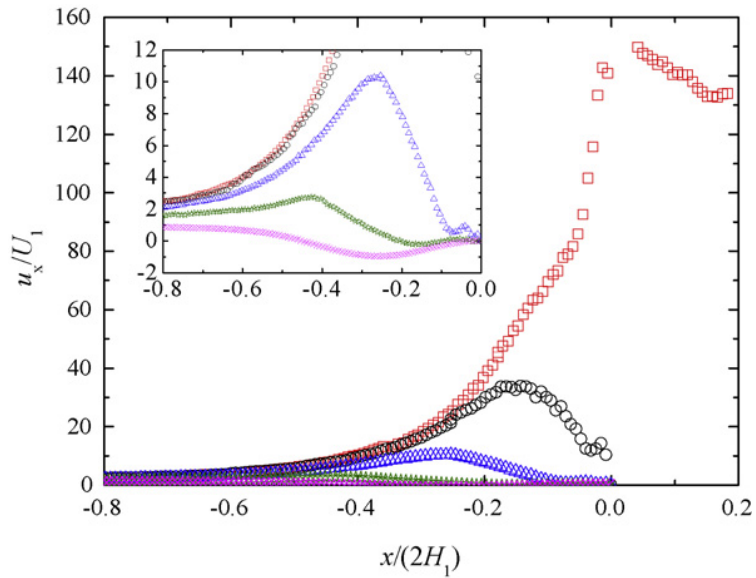

Fig. 19. Axial velocity profiles along the streamwise direction for $D e_{2}=62.3$, $R e_{2}=0.254$ and $C R=8$, at $y=0$ and $z= \pm k H_{1}$ (or $z=0$ and $y= \pm k H_{1}$ ), where ( $\left.\square\right) k=0$; ( $\mathrm{O}) k=0.125 ;(\triangle) k=0.250$; (访) $k=0.500$; and $(\times) k=0.750$.

For low contraction ratios ( $C R=2.4$ and 4$)$, an increase in the flow rate causes diverging streamlines to appear upstream of the contraction (cf. Fig. 13d). This counterintuitive feature, which is related to the extensional behavior of the flow, has also been observed for viscoelastic fluids (both for shear-thinning and Boger fluids) in converging flows with different geometric characteristics (e.g. Refs. [3,31-34]). The interested readers are referred to the works of Cable and Boger [35-37] for a comprehensive illustration of this flow regime and to the work of Alves and Poole [38] for a discussion of this phenomenon and the conditions under which it arises. This diverging pattern of the flow streamlines is accompanied by a significant increase of the vortex length for $D e_{2} \gtrsim 30(C R=4)$ and $D e_{2} \gtrsim 50(C R=2.4)$ (cf. inset of Fig. 17).

For higher contraction ratios ( $C R=8$ and 12), we observe the development of a large lip vortex (as a result of the engulfment of the corner vortex by the lip vortex) with a convex shape that occupies the whole wall from the re-entrant corner to the far corner.
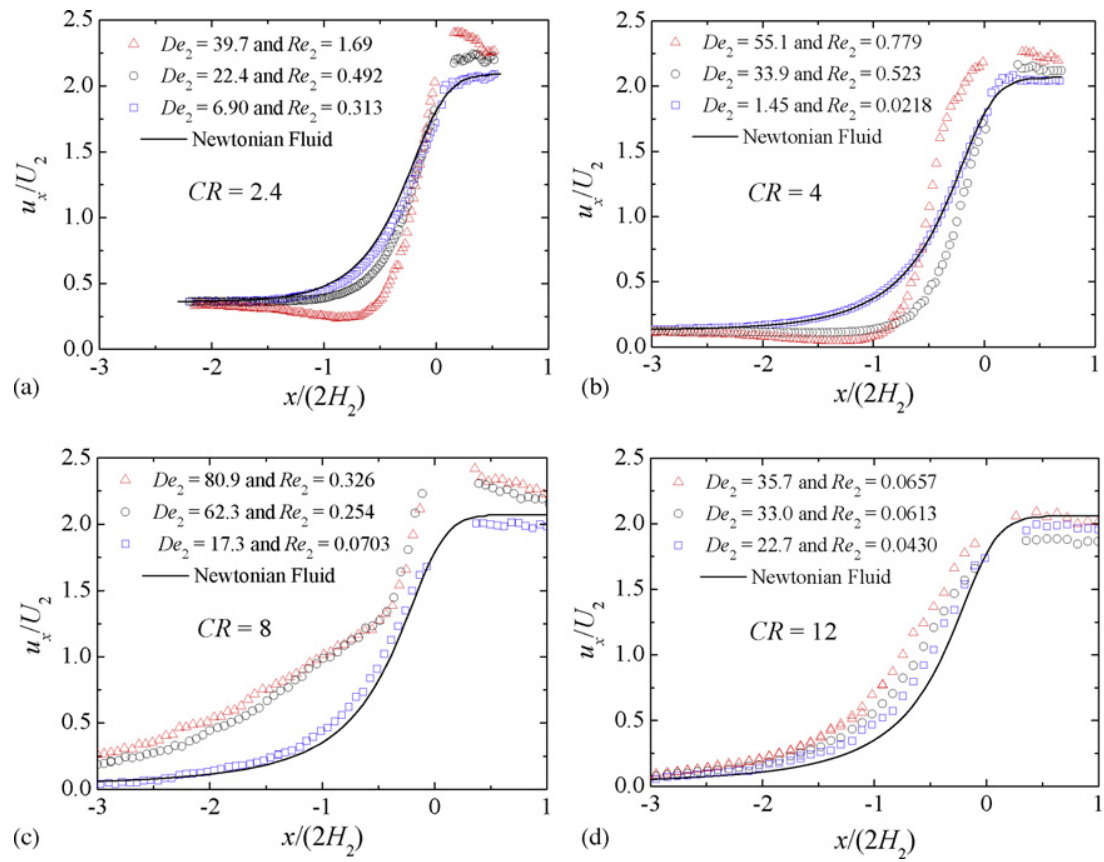

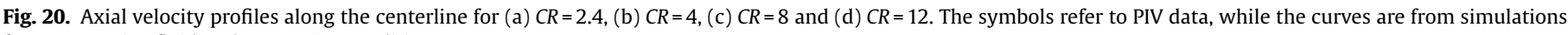
for a Newtonian fluid under creeping conditions. 

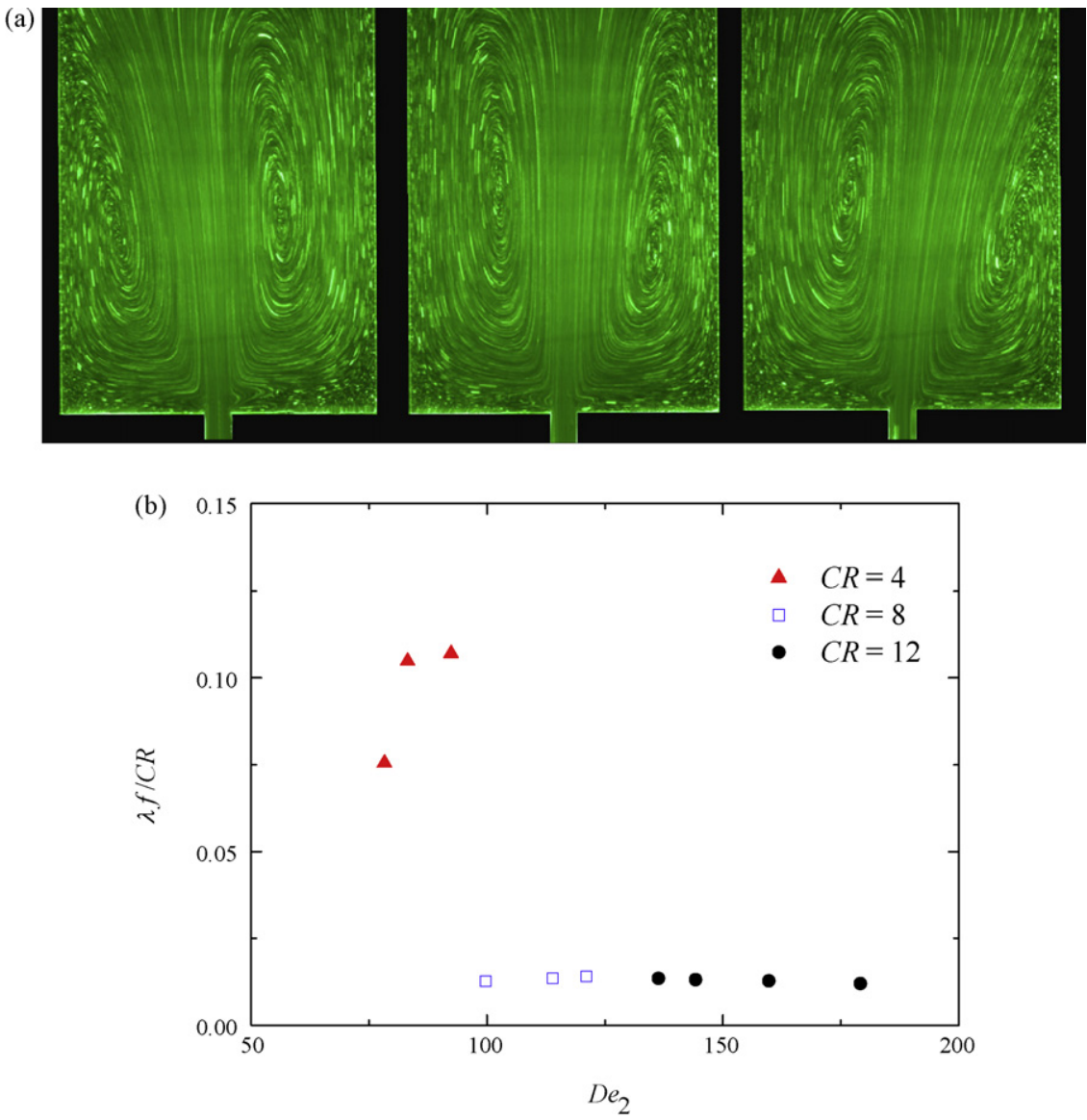

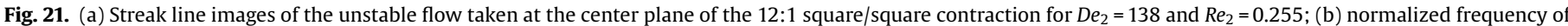
oscillation scaled as a function of the Deborah number for $C R=4, C R=8$ and $C R=12$.

The diverging streamlines seen for low $C R$ are not observed here. However, concurrently with the vortex growth regime, the flow exhibits another remarkable feature $\left(D e_{2} \gtrsim 30\right)$ : the streak lines beneath the main vortical structure close to the re-entrant corner are locally strongly divergent and exhibit a bent-elbow shape (cf. Figs. 14e and 15e) almost as if the fluid had to detour around some small "invisible obstacle". Such sharp divergent streamlines are not observed for lower $C R$, at least not for the range of flow conditions studied. In Fig. 18, we show a zoomed view of this flow feature at the center plane, which includes both results of flow visualization and PIV. Fig. 18a and b shows the velocity field in two different forms, as a contour plot and as a vector plot, respectively. The technique employed is able to capture the flow feature in detail within a large area of interest. To further attest the validity of the PIV measurements we compare the streak lines obtained by streak-line photography (Fig. 18c) and those obtained from integration of the velocity field obtained using PIV (Fig. 18d). Even though the streak lines obtained using the PIV technique are not as smooth near the contraction plane, good agreement is observed between the two techniques.

In Fig. 19, we show the axial velocity profile along the streamwise direction in five different lines $\left(y=0, z=k H_{1}\right.$ or $z=0, y=k H_{1}$ where $k=0,0.125,0.25,0.5$ and 0.75 ) to highlight the sudden axial velocity increase as the fluid moves very close toward the contraction plane $\left(x /\left(2 H_{1}\right) \gtrsim-0.05\right)$ for the planes $z / H_{1}=0.125$ and 0.25 (cf. inset Fig. 19). This characteristic of the velocity profiles, which is not observed for Newtonian-like flow, is a consequence of the presence of localized diverging-converging streamlines in this region. In addition, we highlight the three-dimensionality and symmetry of the flow under these conditions - the profiles for different $z$-planes shown here are in all similar to the profiles in the corresponding $y$-planes.

The effect of the Deborah number on the profiles of the streamwise velocity along the centerline $(y=0$ and $z=0)$ is shown in Fig. 20 for the four contraction ratios studied. At low $D e_{2}$, the profiles resemble those obtained with a Newtonian fluid under creeping flow conditions. Upstream of the contraction, the fluid is seen to accelerate as it approaches the contraction $\left(x /\left(2 H_{1}\right) \gtrsim-0.2\right)$ and the profiles may exhibit a velocity overshoot in the region of the contraction plane $\left(x /\left(2 \mathrm{H}_{1}\right) \approx 0\right)$, that increases significantly with increasing $D e_{2}$. For the lower contraction ratios $(C R=2.4$ and 4$)$, when divergent streamlines are observed a velocity undershoot upstream of the contraction $\left(-0.6 \lesssim x / 2 H_{1} \lesssim 0\right)$ is clearly visible, in agreement with Refs. [38,39]. In Fig. 20 we also include the velocity profile obtained numerically for the Newtonian fluid under creeping flow conditions to highlight that larger $D e_{2}$ and lower $C R$ strengthen the diverging flow and as a result the undershoot effect on the velocity profile is more pronounced.

As $D e_{2}$ increases even further the flow eventually becomes unstable due to an elastic instability (cf. Figs. 13f, 14f and 15f). In this regime, the flow is asymmetric and the vortical structures as a whole are rotating periodically in the azimuthal direction. Additionally, the degree of asymmetry is seen to increase with increasing Deborah numbers. Fig. 21a shows a sequence of streak-line images at the center plane within a cycle of flow oscillation. From films taken with a video camera at a known frame rate we were able to measure the frequency of oscillation, $f$, for different flow rates within the periodic regime. The normalized frequency of this process $(\lambda f / C R)$ was found to be approximately independent of the Deborah number and of the contraction ratio for high $C R$, as shown 


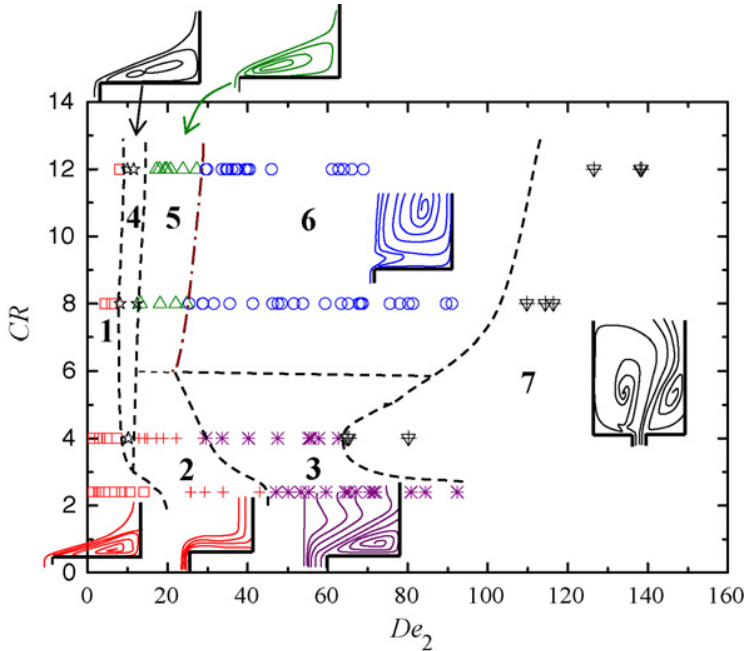

Fig. 22. Flow pattern map. 1, Newtonian-like flow; 2, vortex decrease; 3, vortex growth and upstream diverging streamlines; 4 , simultaneous lip and corner vortices; 5, large lip vortex; 6 , large vortex and localized diverging streamlines at the contraction entrance; and 7 , unstable flow.

in Fig. 21b. In this regime, the diverging streamlines with a bentelbow shape are still present even though they are now skewed by the asymmetry of the flow (cf. Fig. 21a). Analogously to the vortex size results, the frequency results for high $C R$ are consistent with each other but are different from those obtained for lower $C R$ $(C R=4)$.

To summarize, Fig. 22 displays a map of flow patterns and their location on the two-dimensional $C R-D e_{2}$ parameter space. This map is a result of extensive experiments for various contraction ratios and increasing Deborah numbers. The identified flow features are classified as: Newtonian-like flow (1) (Figs. 12a, 13a, 14a and 15a); vortex decrease (2) (Figs. 12b and 13c); vortex growth and upstream diverging stream- lines (3) (Figs. 12e, 12f, 13d and 13e); simultaneous lip and corner vortices (4) (Figs. 13b, 14b and 15b); large lip vortex (5) (Figs. 14c and 15c); large vortex with localized diverging streamlines at the contraction entrance (6) (Figs. 14e and 15e); unstable flow (7) (Figs. 13f, 14f and 15f).

\subsection{Pressure drop}

The pressure drop between the reference port upstream of the contraction and each of the downstream pressure ports were measured experimentally as a function of the flow rate. These results are shown in Fig. 23 and can be useful for benchmarking purposes. The pressure drop vs. flow rate curves display similar characteristics for all $C R$ studied, with the pressure drop increasing non-linearly with the flow rate. Also shown in Fig. 23 is the extra pressure drop ( $\Delta p_{\text {extra }}$ ) caused by the extensional flow in the contraction. The extra pressure drop is usually associated with vortex enhancement in contraction flows and is calculated by subtracting the pressure drop due to fully-developed (fd) Poiseuille flow in the duct upstream $\left(\Delta p_{\mathrm{fd}, \mathrm{u}}\right)$ and downstream $\left(\Delta p_{\mathrm{fd}, \mathrm{d}}\right)$ of the contraction from the total pressure drop $\left(\Delta p_{\text {Total }}\right)$ across the contraction. In our case:

$$
\begin{aligned}
\Delta p_{\text {extra }} & =\Delta p_{\text {Total }}-\Delta p_{\mathrm{fd}, \mathrm{u}}-\Delta p_{\mathrm{fd}, \mathrm{d}} \\
& =\Delta p_{3}-\left|\frac{\mathrm{d} p}{\mathrm{~d} x}\right|_{\mathrm{fd}, \mathrm{u}}\left|\Delta x_{0}\right|-\left|\frac{\mathrm{d} p}{\mathrm{~d} x}\right|_{\mathrm{fd}, \mathrm{d}}\left|\Delta x_{3}\right|
\end{aligned}
$$

where the subscripts ' $u$ ' and ' $d$ ' refer to upstream and downstream ducts, respectively.

We have estimated $\mathrm{d} p / \mathrm{d} x$ in two different ways, one considering the theoretical values of $\mathrm{d} p / \mathrm{d} x$ calculated analytically for fully-developed Poiseuille flow [40] and the other using an experimental value of $\mathrm{d} p / \mathrm{d} x$ determined from measurements at locations 2 and 3: $\mathrm{d} p / \mathrm{d} x=\left(\Delta p_{3}-\Delta p_{2}\right) /\left(\Delta x_{3}-\Delta x_{2}\right)$. For $C R=2.4$ and 4 , we observe a considerable discrepancy between the curves obtained using the two approaches referred above: while the experimental values of $\Delta p_{\text {extra }}$ become negative, which is representative of a local pressure recovery as the fluid goes through the contraction, the esti-
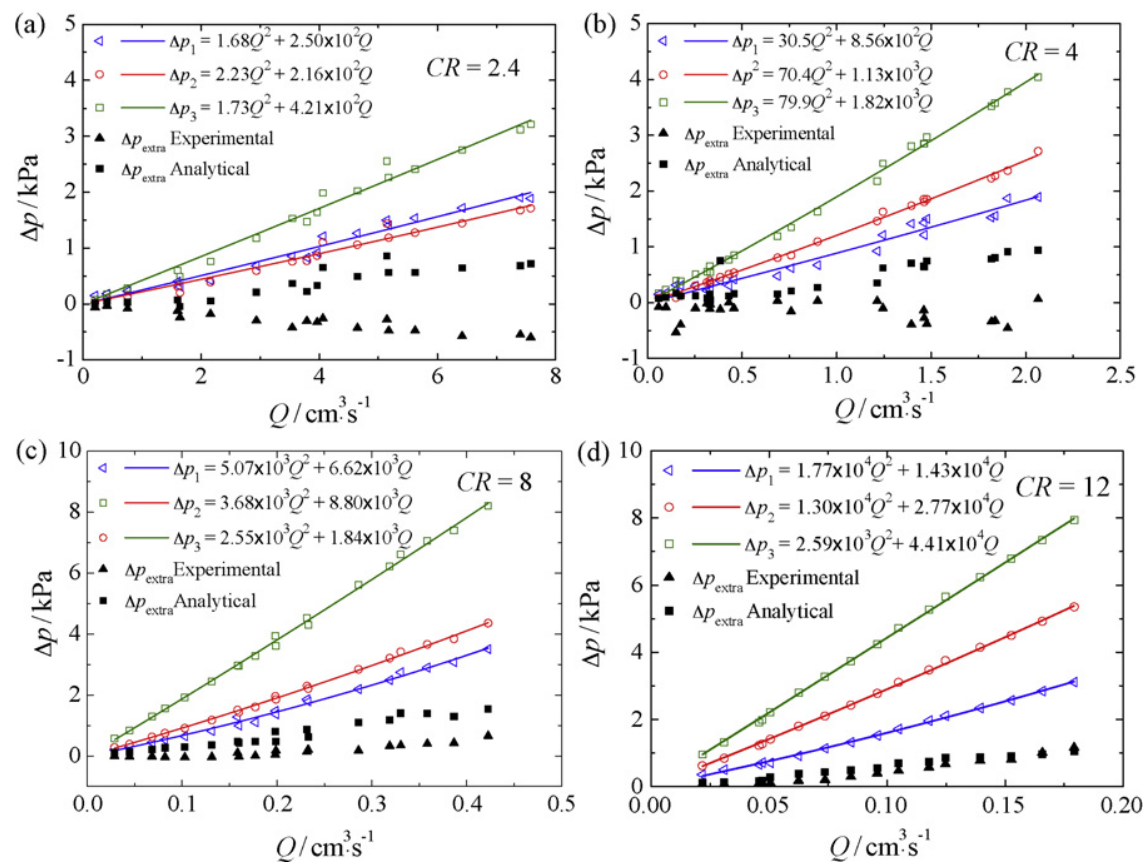

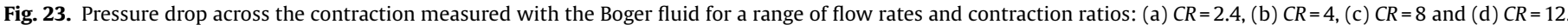

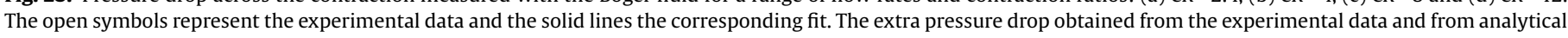
calculations [40] are shown as solid symbols. 


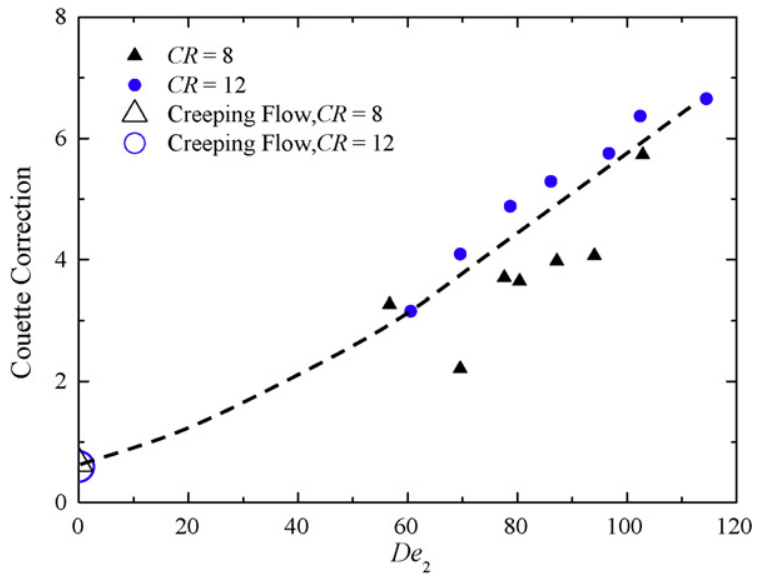

Fig. 24. Couette correction as a function of Deborah number for $C R=8$ and $C R=12$. Also shown are the values of the Couette correction obtained numerically for a Newtonian fluid under creeping conditions $\left(D e_{2} \rightarrow 0\right)$. The dashed curve is a guide to the eye.

mated extra pressure drop from analytical values remain positive and increase with increasing flow rate. In fact, for such $C R$ the extra pressure drop is very small compared to the total pressure drop and therefore a large degree of uncertainty is associated with the estimation of $\Delta p_{\text {extra }}$ since the extra pressure drop is estimated from extrapolation of the ideal (fully-developed) pressure profile starting at a distant pressure location (to guarantee that fully-developed conditions are observed). Additionally, at the locations considered for the measurements the flow might not yet be fully developed (cf. Fig. 20a and b). For higher contraction ratios ( $C R=8$ and 12), the differences between the two approaches diminish significantly and in particular for $C R=12$ the results obtained using the two methods are nearly identical, an indication that for this $C R$ the determination of the entry pressure drop has reasonable accuracy.

The extra pressure drop is typically reported in dimensionless form as a Couette correction, defined as $C=\Delta p_{\text {extra }} / 2 \tau_{w}$, where $\tau_{w}$ is the average downstream fully-developed wall shear stress, which for a square duct can be estimated as $(-\mathrm{d} p / \mathrm{d} x)_{\mathrm{fd}, \mathrm{d}} \mathrm{H}_{2} / 2$. The evolution of the Couette correction with the Deborah number in the range $60<D e_{2}<120$ is shown in Fig. 24 for the higher contraction ratios studied ( $C R=8$ and 12 ), illustrating a significant increase of the Couette correction with $D e_{2}$. Similarly to what was found for the vortex length, the results for $C R=8$ and 12 are identical. We should emphasize that this parameter is difficult to estimate experimentally since it requires the flow to be fully-developed, the measurements have to be performed far downstream of the contraction and therefore any small error on the estimation of the slope $(\mathrm{d} p / \mathrm{d} x)_{\mathrm{fd}, \mathrm{d}}$ will have a strong impact on the extrapolated pressure values and consequently on the extra pressure drop and Couette correction.

\section{Conclusions}

The flow through sudden square-square contractions was characterized experimentally using streak-line photography, pressure drop measurements and PIV, which can be valuable for benchmarking purposes. Two fluids with different rheological properties were used: a Newtonian fluid and a Boger fluid. Additionally, the effect of the contraction ratio was investigated $(C R=2.4,4,8$ and 12$)$. For the Newtonian fluid, in addition to the experimental measurements, numerical simulations were carried out using a finite volume code. The experimental results of flow pattern and velocity field were found to be in good agreement with the numerical results for the whole range of conditions tested, validating the experimental techniques.
By taking advantage of the visualizations of the flow patterns and quantification of the velocity field at various planes along the spanwise directions ( $y$ or $z$ ) covering the whole distance from the wall to the center plane, we have shown that the flow is highly three-dimensional with open vortex structures forming upstream of the contraction. Furthermore, we have demonstrated that the dynamics inside the open recirculations for the Boger fluid at high Deborah numbers are reversed relative to Newtonian fluid flow. For the viscoelastic fluid, tracer particles that enter the recirculations from the upstream duct at the center plane exit at the diagonal plane towards the downstream duct while for the Newtonian fluid, the opposite process takes place with fluid elements entering through the diagonal plane and exiting at the center plane close to the re-entrant corner. For most conditions tested, the flow was found to be symmetrical relative to the diagonal planes $(y= \pm z)$ as well as to the $y$-plane and $z$-plane. However, in the case of the Boger fluid, the flow becomes time dependent for high Deborah numbers and in this regime, the flow loses its overall symmetry.

For the Newtonian fluid, the size of the corner vortex that forms upstream of the contraction was found to depend not only on the Reynolds number but also on the contraction ratio of the geometry. Under low inertial flow conditions, the size of the corner vortex is nearly independent of $R e$ but as $R e$ is increased, a reduction of vortex size is observed. For the Boger fluid, the vortex size was seen to depend on $D e_{2}$ and $C R$ in a complex way, showing two distinct behaviors according to the contraction ratio:

- For low contraction ratios, the vortex growth regime is preceded by a decrease in vortex size until a minimum size is reached. Approximately beyond this minimum, we observe the onset of divergent streamlines upstream of the contraction, displaying a bell-shaped form in the region just upstream of the corner vortex similarly to observations in other converging geometries. This diverging flow pattern is strengthened for larger $D e_{2}$ and lower $C R$ and results in an undershoot in the axial velocity profiles along the centerline upstream of the contraction entrance. Furthermore, the fluid at the centerline is seen to accelerate and the streamwise velocity profiles exhibit a velocity overshoot in the vicinity to the contraction plane, which is enhanced for increasing $D e_{2}$.

- For high $C R$, a monotonous vortex growth was observed, and the size of the corner vortex is dictated by the scaled Deborah number, $D e_{2} / C R$. For $D e_{2} \gtrsim 30$, an atypical type of divergent flow is observed in which the streamlines are strongly divergent, revealing a bent-elbow shape near the re-entrant corner just beneath the large corner vortex. This atypical divergent flow also causes an overshoot in the axial velocity profiles at the centerline, which becomes more pronounced for higher $C R$. In addition, as a consequence of the locally divergent streamlines, an undershoot is observed in the axial velocity profiles along the spanwise direction at an axial position which intersects the region where the flow is strongly divergent. The extra pressure drop was found to increase significantly with the Deborah number, in line with previous works with axisymmetric contractions.

In summary, when elastic effects become important as $D e_{2}$ is increased, the flow becomes significantly different from that of a Newtonian fluid and we have identified a number of distinct flow type regions which we have mapped in the $C R-D e_{2}$ parameter space. These regions include, amongst others, a region in which lip and corner vortices coexist, two distinct regions of diverging flow which are associated with vortex growth but exhibit different characteristics for low and high contraction ratios, and unstable flow in which the vortex size varies periodically in time. 


\section{Acknowledgements}

The authors acknowledge the financial support provided from Fundação para a Ciência e a Tecnologia (FCT) and FEDER through projects POCI/EQU/59256/2004, PTDC/EME-MFE/70186/2006, REEQ/262/EME-2005 and REEQ/928/EME-2005. P.C. Sousa and M.S.N. Oliveira would also like to thank FCT for financial support through scholarships SFRH/BD/28846/2006 and SFRH/BPD/34141/ 2006, respectively.

\section{References}

[1] D.V. Boger, Viscoelastic flows through contractions, Annu. Rev. Fluid Mech. 19 (1987) 157-182.

[2] S.A. White, A.D. Gotsis, D.G. Baird, Review of the entry flow problem-experimental and numerical, J. Non-Newton. Fluid Mech. 24 (1987) 121-160

[3] D.V. Boger, K. Walters, Rheological Phenomena in Focus, Elsevier, Amsterdam, 1993

[4] S. Nigen, K. Walters, Viscoelastic contraction flows: comparison of axisymmetric and planar configurations, J. Non-Newton. Fluid Mech. 102 (2002) 343-359.

[5] M.A. Alves, P.J. Oliveira, F.T. Pinho, On the effect of contraction ratio in viscoelastic flow through abrupt contraction, J. Non-Newton. Fluid Mech. 122 (2004) 117-130.

[6] M.S.N. Oliveira, P.J. Oliveira, F.T. Pinho, M.A. Alves, Effect of contraction ratio upon viscoelastic flow in contractions: the axisymmetric case, J. Non-Newton. Fluid Mech. 147 (2007) 92-108.

[7] O. Hassager, "Working group on numerical techniques", Fifth International Workshop on Numerical Methods in Non-Newtonian Flows, Lake Arrowhead, USA, J. Non-Newton. Fluid Mech. 29 (1988) 2-5.

[8] R.A. Brown, G.H. McKinley, Report on the VIII International Workshop on Numerical Methods in Viscoelastic Flows, J. Non-Newton. Fluid Mech. 52 (1994) 407-413.

[9] L.E. Rodd, T.P. Scott, D.V. Boger, J.J. Cooper-White, G.H. McKinley, The inertioelastic planar entry flow of low-viscosity elastic fluids in microfabricated geometries, J. Non-Newton. Fluid Mech. 129 (2005) 1-22.

[10] S. Gulati, S.J. Muller, D. Liepmann, Direct measurements of viscoelastic flows of DNA in a 2:1 abrupt planar micro-contraction, J. Non-Newton. Fluid Mech. 155 (2008) 51-66.

[11] M.S.N. Oliveira, L.E. Rodd, G.H. McKinley, M.A. Alves, Simulations of extensional flow in microrheometric devices, Microfluid. Nanofluid. 5 (2008) 809826.

[12] M.S.N. Oliveira, M.A. Alves, G.H. McKinley, F.T. Pinho, Viscous flow through microfabricated hyperbolic contractions, Exp. Fluids 43 (2007) 437-451.

[13] K. Walters, M.F. Webster, On dominating elastico-viscous response in some complex flows, Phil. Trans. R. Soc. Lond. A 308 (1982) 199-218.

[14] S.C. Xue, N. Phan-Thien, R.I. Tanner, Three dimensional numerical simulations of viscoelastic flows through planar contractions, J. Non-Newton. Fluid Mech. 74 (1998) 195-245.

[15] S.C. Xue, N. Phan-Thien, R.I. Tanner, Numerical investigations of Lagrangian unsteady extensional flows of viscoelastic fluids in 3-D rectangular ducts with sudden contractions, Rheol. Acta 37 (1998) 158-169.

[16] G. Mompean, M. Deville, Unsteady finite volume simulation of Oldroyd-B fluid through a three-dimensional planar contraction, J. Non-Newton. Fluid Mech. 72 (1997) 253-279.
[17] G. Mompean, M. Deville, Corrigendum to "Unsteady finite volume simulation of Oldroyd-B fluid through a three-dimensional planar contraction" [J. NonNewton. Fluid Mech. 72 (1997) 253-279], J. Non-Newton. Fluid Mech. 103 (2002) 271-272.

[18] I. Sirakov, A. Ainser, M. Haouche, J. Guillet, Three-dimensional numerical simulation of viscoelastic contraction flows using the Pom-Pom differential constitutive model, J. Non-Newton. Fluid Mech. 126 (2005) 163-173.

[19] M.A. Alves, F.T. Pinho, P.J. Oliveira, Visualizations of Boger fluid flows in a 4:1 square-square contraction, AIChE J. 51 (2005) 2908-2922.

[20] M.A. Alves, F.T. Pinho, P.J. Oliveira, Viscoelastic flow in a 3D square/square contraction: visualizations and simulations, J. Rheol. 52 (2008) 1347-1368.

[21] K. Walters, D.M. Rawlinson, On some contraction flows for Boger fluids, Rheol. Acta 21 (1982) 547-552.

[22] R.D. Keane, R.J. Adrian, Theory of cross-correlation analysis of PIV images, Appl. Sci. Res. 49 (1992) 191-215.

[23] S.T. Wereley, C.D. Meinhart, Second order accurate particle image velocimetry, Exp. Fluids 31 (2001) 258-268.

[24] M.L. Sheely, Glycerol viscosity tables, Ind. Eng. Chem. 24 (1932) 1060-1064

[25] R.B. Bird, R.C. Armstrong, O. Hassager, Dynamics of Polymeric Liquids, vol. 1 : Fluid Dynamics, John Wiley \& Sons, New York, 1987.

[26] G.H. McKinley, Nonlinear dynamics of viscoelastic flows in complex geometries, PhD Thesis, Massachusetts Institute of Technology, Cambridge, USA, 1991.

[27] P.J. Oliveira, F.T. Pinho, G.A. Pinto, Numerical simulation of non-linear elastic flows with a general collocated finite-volume method, J. Non-Newton. Fluid Mech. 79 (1998) 1-43.

[28] M.A. Alves, P.J. Oliveira, F.T. Pinho, A convergent and universally bounded interpolation scheme for the treatment of advection, Int. J. Numer. Methods Fluids 41 (2003) 47-75.

[29] E. Schreck, M. Schäfer, Numerical study of bifurcation in three-dimensional sudden channel expansions, Comput. Fluids 29 (2000) 583-593.

[30] T.P. Chiang, T.W.H. Sheu, S.K. Wang, Side wall effects on the structure of laminar flow over a plane-symmetric sudden expansion, Comput. Fluids 29 (2000) 467-492.

[31] R.E. Evans, K. Walters, Flow characteristics associated with abrupt changes in geometry in the case of highly elastic liquids, J. Non-Newton. Fluid Mech. 20 (1986) 11-29.

[32] R.E. Evans, K. Walters, Further remarks on the lip-vortex mechanism of vortex enhancement in planar contraction flows, J. Non-Newton. Fluid Mech. 32 (1989) 95-105.

[33] D.V. Boger, R.J. Binnington, Circular entry flows of fluid M1, J. Non-Newton. Fluid Mech. 35 (1990) 339-360.

[34] G.H. McKinley, W.P. Raiford, R.A. Brown, R.C. Armstrong, Nonlinear dynamics of viscoelastic flow in axisymmetrical abrupt contractions, J. Fluid Mech. 223 (1991) 411-456.

[35] P.J. Cable, D.V. Boger, A comprehensive experimental investigation of tubular entry flow of viscoelastic fluids. Part I. Vortex characteristics in stable flow, AIChE J. 24 (1978) 868-879.

[36] P.J. Cable, D.V. Boger, A comprehensive experimental investigation of tubular entry flow of viscoelastic fluids. Part II. The velocity fields in stable flow, AIChE J. 24 (1978) 992-999.

[37] P.J. Cable, D.V. Boger, A comprehensive experimental investigation of tubular entry flow of viscoelastic fluids. Part III. Unstable flow, AIChE J. 25 (1979) 152-159.

[38] M.A. Alves, R.J. Poole, Divergent flow in contractions, J. Non-Newton. Fluid Mech. 144 (2007) 140-148.

[39] R.J. Poole, M.A. Alves, Velocity overshoots in gradual contraction flows, J. NonNewton. Fluid Mech. 160 (2009) 47-54.

[40] F.M. White, Fluid Mechanics, 5th ed., McGraw-Hill, Boston, 2003. 